ARTICLE

https://doi.org/10.1038/s41467-021-25367-z

\title{
Tet2 Controls the Responses of $\beta$ cells to Inflammation in Autoimmune Diabetes
}

Jinxiu Rui ${ }^{1}$, Songyan Deng ${ }^{1}$, Ana Luisa Perdigoto (1) ${ }^{1}$, Gerald Ponath$^{2}$, Romy Kursawe ${ }^{3}$, Nathan Lawlor ${ }^{3}$, Tomokazu Sumida (1D 2 ${ }^{2}$, Maya Levine-Ritterman (1D ${ }^{2}$, Michael L. Stitzel (1) ${ }^{3,4}$, David Pitt ${ }^{2}$, Jun Lu ${ }^{5}$ \& Kevan C. Herold (1D ${ }^{1 凶}$

$\beta$ cells may participate and contribute to their own demise during Type 1 diabetes (T1D). Here we report a role of their expression of Tet2 in regulating immune killing. Tet2 is induced in murine and human $\beta$ cells with inflammation but its expression is reduced in surviving $\beta$ cells. Tet2-KO mice that receive WT bone marrow transplants develop insulitis but not diabetes and islet infiltrates do not eliminate $\beta$ cells even though immune cells from the mice can transfer diabetes to $\mathrm{NOD} /$ scid recipients. Tet2-KO recipients are protected from transfer of disease by diabetogenic immune cells.Tet2-KO $\beta$ cells show reduced expression of IFN $\gamma$ induced inflammatory genes that are needed to activate diabetogenic $T$ cells. Here we show that Tet2 regulates pathologic interactions between $\beta$ cells and immune cells and controls damaging inflammatory pathways. Our data suggests that eliminating TET2 in $\beta$ cells may reduce activating pathologic immune cells and killing of $\beta$ cells.

\footnotetext{
${ }^{1}$ Departments of Immunobiology and Internal Medicine, Yale University, New Haven, CT, USA. ${ }^{2}$ Department of Neurology, Yale School of Medicine, New Haven, CT, USA. ${ }^{3}$ The Jackson Laboratory for Genomic Medicine, Farmington, CT, USA. ${ }^{4}$ Department of Genetics and Genome Sciences and Institute for Systems Genomics, University of Connecticut, Farmington, CT, USA. ${ }^{5}$ Department of Genetics, Yale University, New Haven, CT, USA.

凶email: kevan.herold@yale.edu
} 
T ype 1 diabetes (T1D) is a chronic autoimmune condition that occurs over years after the first signs of autoimmunity, which are represented by the appearance of autoantibodies. With the improvement in the sensitivity of measurement of $\mathrm{C}$-peptide and the availability of tissue sections from individuals who have died with T1D, it has become clear that not all $\beta$ cells are destroyed by autoimmunity. Even 50 years after the diagnosis of T1D about $2 / 3$ of patients still have detectable levels of $\mathrm{C}$-peptide indicating residual $\beta$ cells ${ }^{1}$. Moreover, the increased relative levels of proinsulin suggest dysfunctional but persistent $\beta$ cells ${ }^{2}$. The reasons why some $\beta$ cells survive and others succumb to autoimmune killing are not certain.

There is growing evidence showing $\beta$ cells are not passive bystanders of their own destruction, but instead participate and contribute to their own demise in the pathogenesis of autoimmune diabetes. Genome-wide association studies (GWAS) show that $>50 \%$ of gene loci associated with T1D are expressed in $\beta$ cells. These studies, coupled with emerging molecular evidence that $\beta$ cells are impaired by gain-of-function or loss-of-function of these loci, suggest an active role for the $\beta$ cell in eliciting its own demise ${ }^{3-6}$. In addition to the intrinsic features of $\beta$ cells, accumulating data suggest that $\beta$ cells can control pathways of immune tolerance. $\beta$ cells are required to initiate diabetes autoimmunity. Spleen cells from $\beta$-cell-deprived NOD mice cannot transfer diabetes although they maintain immune competency. Furthermore, "diabetogenic" spleen cells showed a reduced capacity to transfer diabetes after transient "parking" in $\beta$-celldeprived mice ${ }^{7}$. The islet cell mass plays a critical role in diabetes - surgical removal of $90 \%$ of pancreatic tissue before the onset of insulitis induced a long-term protection in NOD mice. Lymphocytes from pancreatectomized diabetes-free mice exhibited a low response to islet cells ${ }^{8}$. Curiously, in NOD mice and human T1D, autoimmunity does not extend to cells that share antigens with $\beta$ cells, particularly cohabitating endocrine islet cells and neuronal cells that express GAD and IA-2. It is thus likely that features of $\beta$ cells that cause expression of autoantigens and their presentation under conditions for $\mathrm{T}$ cell activation are prerequisites to the development of autoimmune lesions. Thus, the interplay between $\beta$ cells and immune cells determine the outcome of autoimmunity.

$\beta$ cells are also known to be intrinsically vulnerable to killing, and are more prone than other islet endocrine cells, to death under ER and immune stress conditions ${ }^{9,10}$. Early physiological $\beta$ cell death triggers priming of autoreactive $\mathrm{T}$ cells by dendritic cells in pancreatic lymph nodes $(\mathrm{pLNs})^{11,12}$. Thompson et al. described a subpopulation of $\beta$ cells that become senescent and actively promotes the immune-mediated destruction process ${ }^{13}$. Recently Lee et al. reported that modulating the unfolded protein response (UPR) in NOD $\beta$ cells by deleting the UPR sensor IRE1 $\alpha$ prior to insulitis induced transient dedifferentiation of $\beta$ cells, resulting in substantially reduced islet immune cell infiltration and $\beta$ cell apoptosis and protection from diabetes ${ }^{14}$. When we previously analyzed $\beta$ cells during the progression of diabetes, we identified a subset of $\beta$ cells with dedifferentiated features such as reduced expression of $P d x 1, N k x 6.1$, MafA, and Ins1, Ins2 as well as $\beta$ cell autoantigens, and those cells were resistant to immunemediated killing ${ }^{15}$.

Among the mechanisms that might account for the changes of $\beta$ cells in response to stressors are epigenetic changes involving silencing or activation of genes as a consequence of signaling by factors such as inflammatory cytokines. Stefan-Lifshitz et al. associated DNA hydroxymethylation by IFNa ${ }^{16}$. In our previous studies, we found epigenetic changes in $\beta$ cells from NOD mice that led to methylation marks in Ins1 and Ins2 and reduced gene transcription and similar changes in INS when human islets were cultured with inflammatory cytokines ${ }^{17}$. The increased expression of Dnmts, we postulated resulted in methylation of $\mathrm{CpG}$ sites in the insulin genes and repression of gene transcription ${ }^{17}$.

The responses to inflammation and epigenetic modifications may be linked by the activity of the ten-eleven translocation (Tet) methylcytosine dioxygenase family members. Zhang et al. showed that Tet2 is required to resolve inflammation by recruiting Hdac2 to repress IL-6. In melanoma and colon tumor cells deletion of Tet2 reduced chemokine expression and tumor-infiltrating lymphocytes, enabling tumors to evade antitumor immunity and to resist anti-PD-L1 therapy ${ }^{18}$. Whereas enhancing TET activity with ascorbate/vitamin $\mathrm{C}$ increased chemokines, tumorinfiltrating lymphocytes, and antitumor immunity ${ }^{18,19}$. In primary microglial cells lacking Tet2, there is inhibition of IL-6 release and TNF- $\alpha$ after LPS treatment ${ }^{20}$. Deletion of Tet2 in $\mathrm{T}$ cells decreased their cytokine expression, associated with reduced p300 recruitment ${ }^{21}$. Lastly, a role for Tet 2 in cell survival has also been suggested as Tet2-KO hematopoietic stem and progenitor cells have reduced apoptosis ${ }^{22}$.

To understand how epigenetic modifications may change $\beta$ cells and affect immune/ $\beta$ cell interactions during autoimmune diabetes, we studied the role of Tet2 and epigenetic changes in human and murine $\beta$ cells during the autoimmune attack. Here we show that Tet2 regulates pathologic interactions between $\beta$ cells and immune cells in humans with immune infiltrates in the pancreas and with diabetes and in mice with autoimmune diabetes by controlling damaging inflammatory pathways.

\section{Results}

Induction of Tet 2 in murine $\beta$ cells during the progression of autoimmune diabetes. We first studied Tet gene expression in whole islets and $\beta$ cells from NOD mice, a model of human T1D. Soon after weaning, at 3-4 weeks, a period of time that has been associated with the initiation of autoimmunity, we found a modest increase in expression of Tet 1 but substantially increased expression of Tet2 in whole islets (Fig. 1a) in NOD mice but not immune-deficient NOD/scid mice of the same age. We identified these changes in Tet genes in $\beta$ cells from 8- and 11-week-old NOD mice by enriching for $\beta$ cells by sorting on Zinc + and TMRE + islet cells. (Fig. 1b). Because of the profound increase in Tet2 expression in NOD $\beta$ cells, we focused our studies on this epigenetic regulator. We examined individual inflammatory cytokine as well as cytokine combinations which have been found to mediate $\beta$ cell killing during the pathogenesis of autoimmune diabetes, on Tet 2 induction in islets from B6 mice ${ }^{23}$. IL-1 $1 \beta$ or IFN $\gamma$ alone and combinations of IL-6/IL-17A and TNF $\alpha /$ IL-1 $\beta /$ IFN $\gamma$ could induce Tet 2 gene transcription in the islets and $\beta$ cells (Fig. 1c) as well as increased protein expression, identified by FACS (Fig. 1d).

Expression of TET2 in human $\boldsymbol{\beta}$ cells. We analyzed TET2 expression in human islets in different inflammatory settings by immunohistochemistry. In control pancreases, TET2 expression was seen outside of the islets among exocrine tissue (Fig. $2 \mathrm{a}(\mathrm{a})$ ). However, there was increased TET2 expression in the nuclear and cytoplasm in $\beta$ cells from a patient with autoimmune pancreatitis (with cellular immune infiltrates) (Fig. 2a(b)), as well as a patient with T1D autoantibodies but not diagnosed with T1D (Fig. 2a(c)), and patients with recent-onset T1D (Fig. 2a(d)). The expression of TET2 in $\beta$ cells was associated with inflammatory lesions since we did not identify it in the $\beta$ cells in the pancreas from patients with chronic pancreatitis in which infiltrating immune cells were not found (Fig. 2a(f)). Interestingly, we also did not identify TET2 staining in the remaining singular $\beta$ cells in the pancreas from patients with long-established T1D who did not have detectable autoantibodies or insulitis (Fig. $2 \mathrm{a}(\mathrm{e})$ ). The 
a

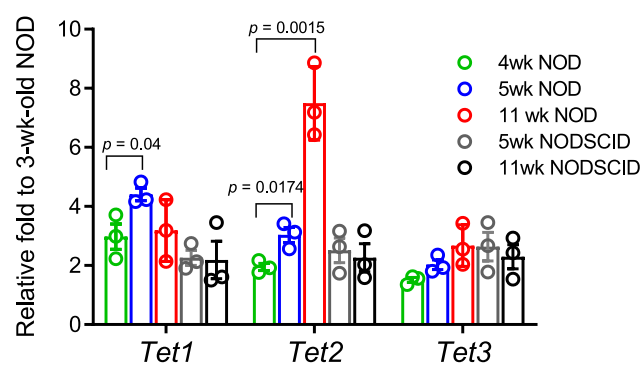

C

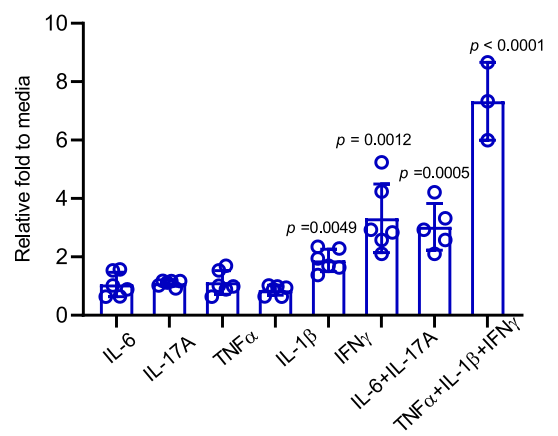

b

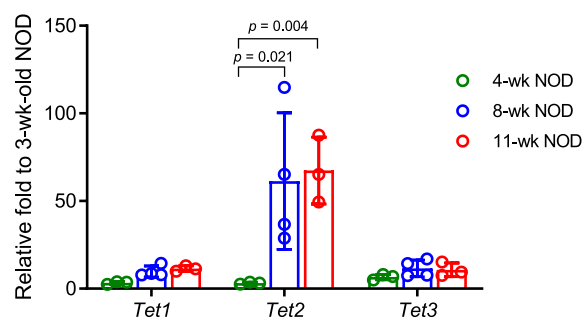

d

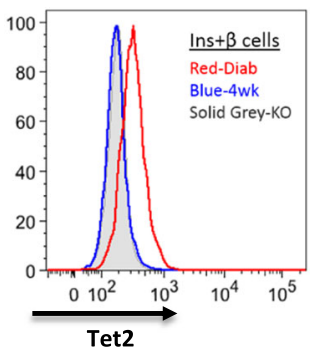

Fig. 1 Increased Tet2 expression in islet $\boldsymbol{\beta}$ cells during diabetes progression in nonobese diabetic (NOD) mice. Transcription analysis of the Tet genes in $\mathbf{a}$ handpicked islets from NOD and NOD/scid mice of different ages, $\mathbf{b}$ enriched $\beta$ cells ( $\mathrm{Zn}+\mathrm{TMRE}+$ ) sorted from NOD mice of different ages, and $\mathbf{c}$ islets from $\mathrm{B} 6$ mice following 24-h culture with indicated cytokines, $10 \mathrm{ng} / \mathrm{ml}$ each. RNA was recovered and Tet genes were measured by qRT-PCR. The Ct values were normalized to $A c t b$ mRNA levels (Delta $C t=(C t$ of $A c t b-C t$ of target gene) +20$)$. Data show the mean $\pm S D$ of three experiments, each with 3-6 mice. Statistical analysis was performed using two-way ANOVA with Tukey's multiple comparisons test in a, b and one-way ANOVA with Dunnett's multiple comparisons test in c. d Histogram comparing Tet2 protein level in $\beta$ cells from 4-week-old as well as new-onset diabetic NOD mice analyzed by FACS. $\beta$ cells from NOD Tet2-knock out (KO) mice were included as a negative control for Tet 2 staining. Tet $2+\beta$ cells were identified by intracellular staining with antibodies against insulin and Tet2. Data were representative of three experiments, $n=3-5$ for each experiment. (Gray histogram $=\beta$ cells from NOD KO mice, Blue = wild-type NOD mice at 4 weeks of age and red = at diagnosis with hyperglycemia).

fluorescence intensity of TET2 staining in $\beta$ cells in different clinical conditions was quantified in Fig. 2b. The expression of TET2 in $\beta$ cells in autoimmune pancreatitis and T1D onset plus some autoantibody + individuals was significantly higher compared to conditions without inflammation. Our findings were consistent with our studies with murine islets and suggested that inflammatory cytokines could induce TET2 expression. We, therefore, analyzed TET2 expression in human islets (Supplementary Table 1) that were cultured with individual cytokine or cytokine combinations and found increased TET2 gene expression in IL-1 $\beta$ or IFN $\gamma$ alone as well as IL6/IL-17A and TNFa/IL$1 \beta / \mathrm{IFN} \gamma$ combinations group (Fig. $2 \mathrm{c}$ ).

These observations suggest that TET2 expression is induced by inflammatory cytokines and suggest a permissive but not sufficient role for $\beta$ cell killing since we found increased TET2 in $\beta$ cells from patients with autoimmune pancreatitis that do not develop diabetes. However, we noted that not all of the $\beta$ cells in patients with T1D or in the autoantibody + relatives were TET2 +. Moreover, in samples from patients with long-standing T1D, we identified $\beta$ cells by insulin staining, consistent with reports of residual $\beta$ cells even in long-standing patients, but we did not find TET2 expression in those $\beta$ cells (Fig. $2 \mathrm{a}(\mathrm{e})$ ).

Resistance of Tet 2 -KO $\boldsymbol{\beta}$ cells to immune killing. To understand the role of Tet 2 in the inflammatory responses of $\beta$ cells, we bred Tet2-KO NOD mice. Tet proteins are known to play an important role in cell development ${ }^{24}$ and Dhawan et al. reported that deletion of Tet 2 in the pancreatic lineage results in improved glucose tolerance and $\beta$ cell function (https://doi.org/10.2337/ db18-50-OR). Therefore, we first characterized $\beta$ cell function, islet cells, and gene expression from Tet2-KO B6 mice. The glucose tolerance to IPGTTs and morphology of the islets were indistinguishable between the $\mathrm{KO}$ and wild-type (WT) mice (Supplementary Fig. 1a, b). There were small but significant differences in the proportion of $\beta$ and $\alpha$ cells in the $\mathrm{KO}$ mice as well as in the MFI of insulin and Pdx1 but the expression of Ins1, Ins2, Nkx6.1, ChgA, and $P d x 1$ were similar to WT $\beta$ cells (Supplementary Fig 1c-e). Overall these findings suggest that there may be subtle differences in the composition of islets from $\mathrm{KO}$ and WT mice but not detectable functional abnormalities of Tet2KO $\beta$ cells.

We backcrossed the KO allele to NOD for more than 14 generations. The Tet2-KO NOD mice did not develop spontaneous diabetes whereas the median time to diabetes was 17 and 23 weeks in their WT and HET littermates (Supplementary Fig. 2).

Because Tet2 is involved in the development and function of immune cells, we transplanted bone marrow (BM) from WT NOD mice into WT or Tet2-KO recipients to eliminate any impact of Tet 2 loss in immune cells. The Tet2-KO mice had a markedly reduced incidence of diabetes (median survival undefined) compared to WT recipients of NOD WT BM (median survival $=11$ weeks $)(p<0.0001)$ (Fig. 3a). This was not due to the lack of immune infiltration in $\mathrm{KO}$ recipients. Figure $3 \mathrm{~b}$ shows the frequencies of immune and nonimmune (CD45+ and -, respectively) from BMT recipients ages 8 to 20 weeks. We assessed the insulitis from KO BMT recipients at 20 weeks and analyzed 112 islets using a 4 point score (0-3). Forty-eight islets are score $0(43 \%), 25$ are score $1(22 \%), 26$ are score $2(23 \%)$, and 13 are score $3(17 \%)$. At the same level of immune cell infiltrates, 
b

a
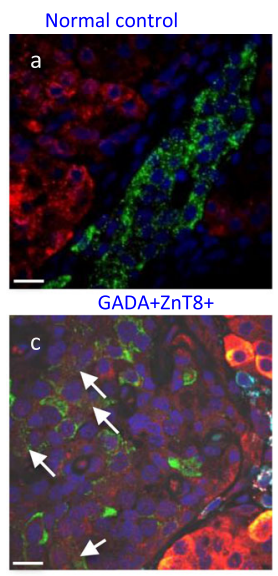

T1D 15yr-Ab negative

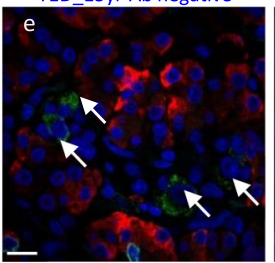

TET2/INS/DAPI/CD45
Autoimmune pancreatitis
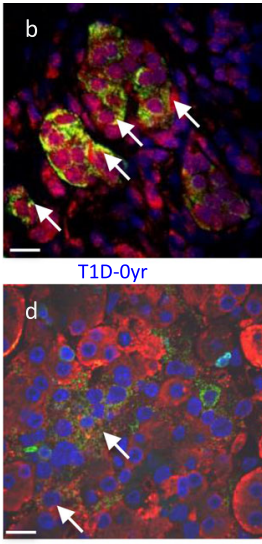

Chronic pancreatitis

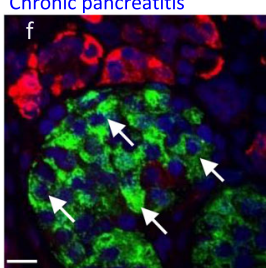

TET/
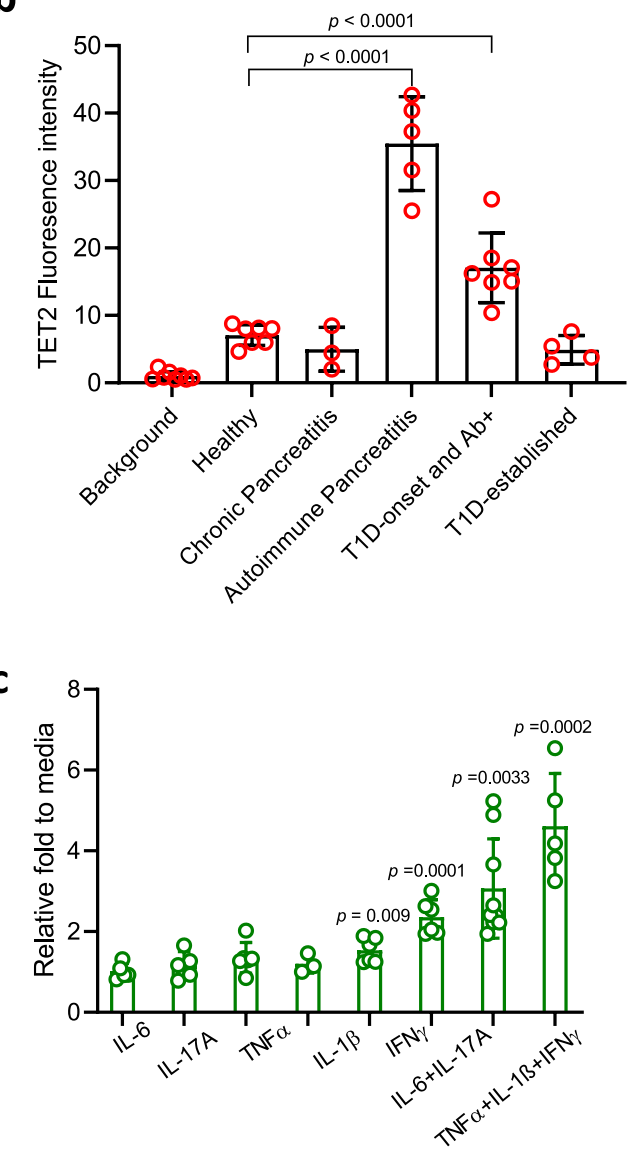

Fig. 2 Induction of TET2 in human $\boldsymbol{\beta}$ cells with inflammation. a Expression of TET2 protein in human $\beta$ cells in vivo in pancreas from a healthy control subject (a), patient with autoimmune pancreatitis (b), a nondiabetic individual with autoantibodies to GADA and ZnT8 who is a relative of a patient with Type 1 diabetes (c), a patient with recent-onset T1D (d), patient with Type 1 diabetes for 15 years without detectable autoantibodies (e), and (f) a patient with chronic pancreatitis. The sections were stained with anti-TET2 (red) and anti-insulin (green) as well as anti-CD45 (Cyan) antibody. DAPI (blue) stains the cell nucleus. The merged staining is shown. Arrows indicate TET $2+\beta$ cells in $b, c$, and $d$ and $\beta$ cells in e and f. Data were from normal individuals $(n=8)$, donors with autoimmune pancreatitis $(n=5)$, nondiabetic donors who were autoantibody $+(n=7)$, and C-peptide + patients with T1D of relatively short duration $(n=7)$. Scale bars: $25 \mu \mathrm{m}$. b Image J quantification of the fluorescence intensity of TET2 staining in islets studied in Fig. 1a. Twenty islets per condition were looked at, with three-seven donors for each condition. Different clinical conditions were compared to a healthy individual. Statistical analysis was performed using one-way ANOVA with Dunnett's multiple comparisons test. c Human islets were cultured with individual or combined cytokines as indicated for $24 \mathrm{~h}$ before transcription analysis of TET2 gene by qRT-PCR. The fold of TET2 mRNA induced by cytokines relative to islets cultured in media alone is shown. Data were mean \pm SD from five experiments, each with 2000-8000 islet equivalents (IEQ) from nondiabetic individuals (Fold vs islets cultured in media alone. Statistical analysis was performed using one-way ANOVA with Dunnett's multiple comparisons test.

there were fewer surviving $\beta$ cells in the WT recipients $(r=-0.82, p<0.0001)$ that was not seen in the Tet2-KO recipients $(p=0.1447)$. It was possible that autoreactive cell development was impaired in the Tet2-KO recipients. However, we found a similar frequency of IGRP-reactive CD8 $+\mathrm{T}$ cells in the WT and Tet2-KO BM recipients (Fig. 3c). In addition, we transferred splenocytes from KO bone marrow transfer (BMT) recipients that did not have diabetes, 11-14 weeks after the BMT, or from diabetic WT BMT recipients, into NOD/scid mice. These $\mathrm{NOD} /$ scid recipients developed diabetes at a similar rate (Fig. $3 \mathrm{~d}$ ), indicating that autoimmune $\mathrm{T}$ cells are present in nondiabetic Tet2-KO BMT recipients.

To further understand the nature of the resistance of $\beta$ cells to killing, we transplanted islets from 5-6-week-old KO vs WT mice under the kidney capsules of 6-week-old NOD WT recipients. Two weeks after the transplant, and in addition, gave $1 \times 10^{7}$ diabetogenic splenocytes to synchronize the development of diabetes. Recipients were followed for the development of hyperglycemia which required the killing of both the endogenous and the transplanted islets. The time to diabetes onset in the recipients of $\mathrm{KO}$ islets was delayed by 4.5 weeks (median survival $=19.5$ weeks) compared to recipients of NOD WT islets (median survival $=15$ weeks) $(p<0.0001)$. All of the 11 recipients of WT islets developed diabetes whereas, at 25 weeks, three of the 12 recipients of KO islets were diabetes-free (Fig. 3e). When the graft-bearing kidney was removed, there was rapid hyperglycemia, indicating that the transplanted islets were the source of insulin in the mice and that the endogenous WT $\beta$ cells in the recipients had been destroyed. The Tet $2-\mathrm{KO} \beta$ cells could be killed by chemical means (i.e., streptozotocin) in vivo (Supplementary Fig. 3), suggesting that the resistance of the $\mathrm{KO} \beta$ cells to killing was to immune mechanisms.

To directly assess the effects of inflammatory mediators on Tet 2 -KO $\beta$ cells, we cultured single islet cells with inflammatory cytokines that cause $\beta$ cell death and found increased survival of Tet $2-\mathrm{KO}$ vs WT $\beta$ cells (Fig. 3f). The resistance of the KO $\beta$ cells was greater than $\alpha$ cells (Fig. 3f). Second, we cultured WT NOD/ scid and Tet2-KO $\beta$ cells with purified islet infiltrates from prediabetic WT NOD mice and found the greater killing of the WT vs Tet2-KO $\beta$ cells (Fig. $3 g$ ). 

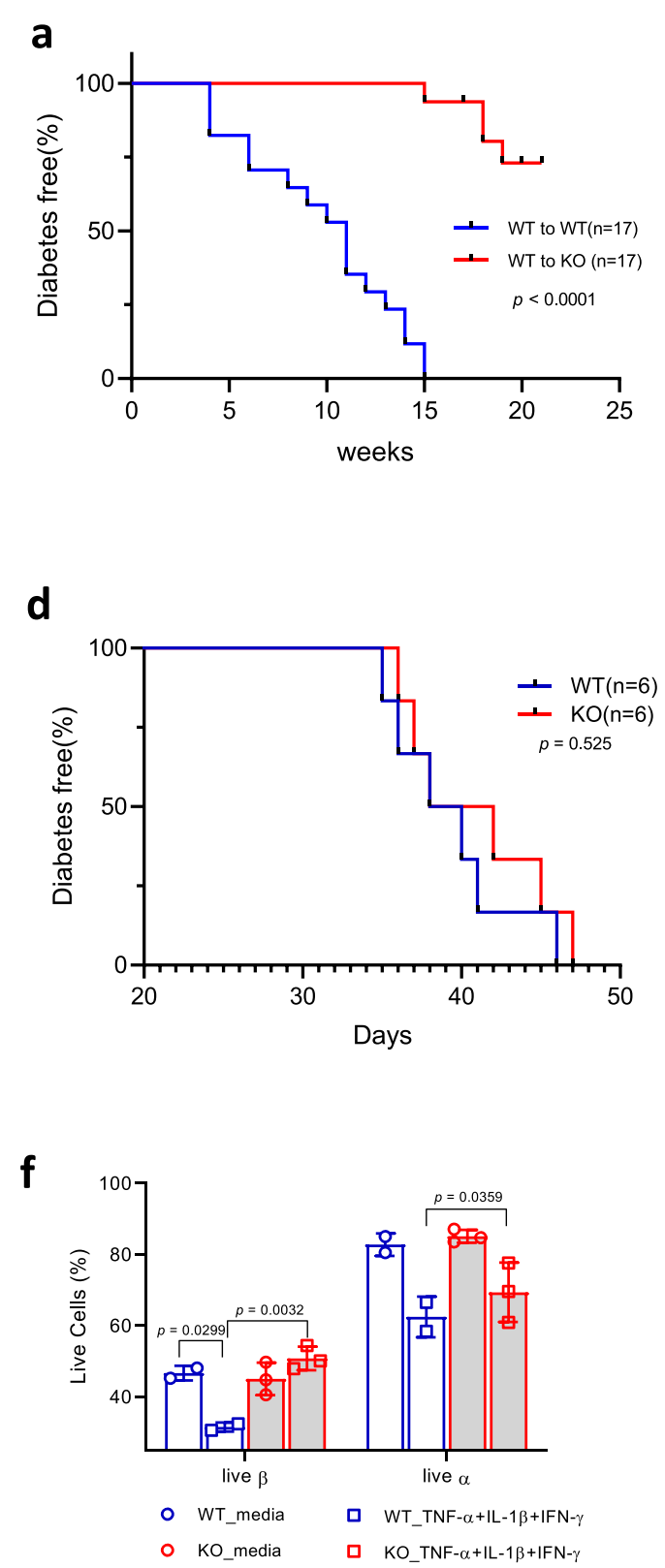

b

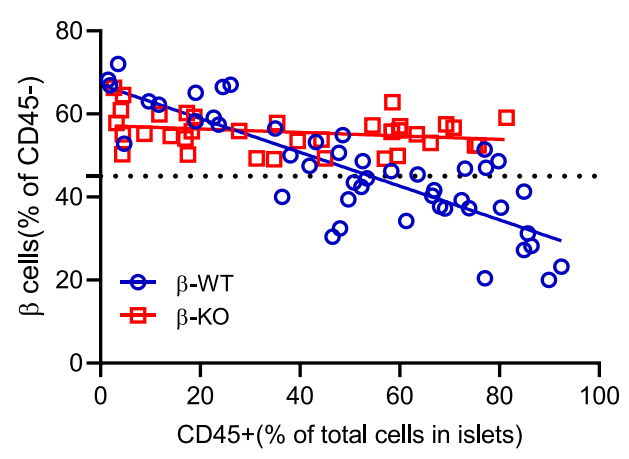

C

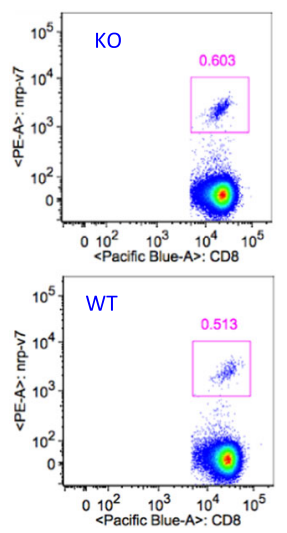

e
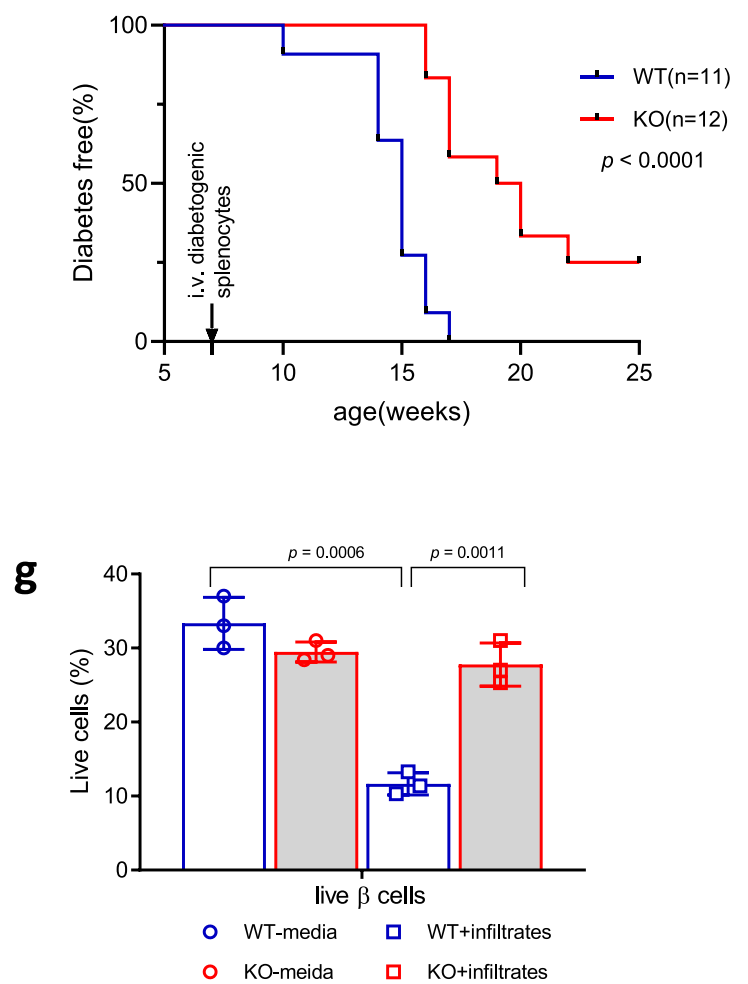

Fig. $3 \beta$ cells lacking Tet2 are protected from immune killing in vivo and in vitro. a Diabetes incidence in WT or KO recipients of WT bone marrow. Statistical analysis was performed using Log-rank curve comparison. $n=17$ for each group. $\mathbf{b}$ Relationship between cellular infiltrates and loss of $\beta$ cells in WT and Tet2-KO recipients of WT bone marrow. The frequency of the intra-islet CD $45+$ cells and $\beta$ cells (insulin + ) analysed by FACS. Simple linear regression was used together with a two-side correlation test for statistical analysis. For WT recipients, Pearson $r=-0.82, p<0.0001$; for Tet2-KO recipients, $p=n s$. Each circle/square represents one mouse (WT, $n=47 ; K O, n=36)$. c Frequency of IGRP-reactive CD8 + T cells in the spleen analysed by FACS. Fourteen weeks after transplant, splenocytes from KO or WT BMT recipients were stained with NRPV7 tetramer to identify IGRP-reactive CD8 + T cells. A single pair, representative of three experiments are shown. $\mathbf{d}$ Transfer of diabetes into NOD/scid mice with $10^{7}$ splenocytes from either nondiabetic KO or hyperglycemic WT BMT recipients 12 weeks post-BMT. Data were from two experiments, each with pooled splenocytes from two KO and two WT BMT recipients. Statistical analysis was performed using Log-rank curve comparison. e Diabetes incidence in islet transplant recipients from either WT or Tet2-KO NOD. About 250-300 pooled islets from 5-6-week-old WT or KO NOD mice were transplanted under the kidney capsule of 6week-old WT NOD followed by transfer of $10^{7}$ diabetogenic splenocytes. The median survival time in WT is 15 vs 19.5 weeks in KO recipients. Statistical analysis was performed using Log-rank curve comparison (WT, $n=11 ; K O, n=12$ ). $\mathbf{f}$ Islet $\beta$ cell survival during culture with cytokines. Single islet cells from WT or Tet2-KO B6 mice were cultured with the indicated cytokines for $12 \mathrm{~h}$ and the live cell percentage was determined in $\beta$ as well as $\alpha$ cells (identified by insulin and glucagon staining) by FACS. Results are mean \pm SD of three experiments, $n=6$ each. Statistical analysis was performed using two-way ANOVA with Tukey's multiple comparisons test. $\mathbf{g} \beta$ cell survival during culture with diabetogenic islets infiltrates. Sorted $\beta$ cells from either WT NOD/scid or Tet2KO NOD mice were cultured overnight with islets infiltrates sorted from the same prediabetic WT NOD at a ratio of 1:5. Results are mean \pm SD of three experiments, $n=5-6$ each. Statistical analysis was performed using one-way ANOVA with Dunnett's multiple comparisons test. 
These initial observations suggested that a subpopulation of $\beta$ cells that survive immune attack have reduced Tet 2 despite the general increase in expression that we had observed during disease progression in vivo, and in human $\beta$ cells that were exposed to inflammatory cytokines. In our previous studies in NOD mice, we identified a subpopulation of $\beta$ cells, characterized by reduced insulin granularity, increased expression of "stemlike" genes, and reduced $\beta$ cell markers such as $P d x 1, N k x 6.1$, and MafA that was resistant to immune-mediated killing ${ }^{15}$. Therefore, we analyzed Tet 2 expression among these subpopulations of $\beta$ cells from NOD mice. We found that Tet 2 levels were higher in the normal (i.e., "top") $\beta$ cells that we showed succumbed to immune killing during the progression of the autoimmune disease when compared to the hypogranular (i.e., "bottom") cells that survived cytokine and immune cell-mediated killing (Supplementary Fig. 4).

Immune responses are modulated by Tet2-deficient islet cells. We analyzed the islet infiltrates from the WT and KO recipients of WT BM, 8 weeks after transplant to understand how the Tet2$\mathrm{KO}$ islet cells changed the immune responses. The frequency of infiltrating cells was similar in the KO and WT islets (Supplementary Fig. 5). We analyzed gene expression by NanoString (pan-immunology panel) and found differential expression of 189 of 770 genes. There was decreased expression of genes associated with kinase signaling needed for $\mathrm{T}$ cell activation such as Stat3, Jak2, Smad3, Nfkb, and Mapk, reduced expression of molecules associated with cell-cell interaction, as well as T cell function such as Cd137, Gzmm, and Gzmk in addition to a panel of reduced cytokines (Fig. 4a). In addition, the infiltrating cells from KO recipients show increased expression of pro-cell death genes such as Bax, Casp3, Casp8, and Tnfsf10 (Trail) and reduced prosurvival gene $\mathrm{Bcl}$ 2. Pathway analysis (with Ingenuity Pathway Analysis (IPA)) identified differences in inflammatory responses implicating 91 genes. Other affected pathways included cell-tocell signaling and interaction, cellular function and maintenance, growth and proliferation, and movement which were significantly decreased in immune cells from KO recipients. Th1 and Th2 activation pathways were reduced in $\mathrm{KO}$ recipients as well as pathways of cell trafficking (Table 1). There were phenotypic difference between the infiltrating $\mathrm{T}$ cells in the KO and WT mice. To confirm these findings, we analyzed immune cells from the draining (pancreatic) lymph nodes from KO and WT BMT recipients of WT BM by FACS. The $\mathrm{T}$ cells from the $\mathrm{KO}$ recipients showed reduced frequencies of total $\mathrm{T}$ cells and increased frequency of $\mathrm{B}$ cells (Fig. $4 \mathrm{~b}$ ). In addition, there was reduced expression of CCR7+ and CXCR3 + CD4 T and CD8 T cells and fewer central memory CD4+ $\mathrm{T}$ cells (Fig. 4c), suggesting that pathologic (i.e., CXCR3+) $\mathrm{T}$ cells are not recruited in the $\mathrm{KO}$ recipients. On diabetes antigen-(IGRP) specific $\mathrm{T}$ cells in the pLNs (Fig. 3c), we likewise found reduced expression of CXCR3. Other activation markers like CD44 and PD1 were similar between $\mathrm{KO}$ vs WT recipients (Fig. $4 \mathrm{~d}$ ).

$\beta$ cells lacking Tet 2 show reduced inflammatory responses. The differences in WT and KO $\beta$ cells might affect the infiltrating immune cells. Therefore we compared $\beta$ cells $(\mathrm{Zn}+\mathrm{TMRE}+)$ from the WT or KO BMT recipients 8 weeks after WT transplant and analyzed the cells by RNA-seq. About 333 genes were found to be differentially expressed between $\mathrm{KO}$ and WT $\beta$ cells $(p<0.05$ after FDR correction (Supplementary Fig. 6)), and 199 of these genes showed $\geq 1.5$-fold change in expression (Fig. 5a, b). We identified a number of differences in pathways associated with $\beta$ cell death and immune protection. These included reduced PTEN signaling $(p=0.00129)^{25,26}$, inflammatory mediators (Irf8 and Tifa), attenuated immune signaling (Pik3cd and Abl2), and cytokine-inducible genes (Gbp2, Gbp6, Ifi47, and Tnfaip2). Genes involved in Class I and II MHC expression were reduced but the levels of Class I MHC were not reduced by FACS on $\beta$ cells from Tet2-KO mice (Supplementary Fig. 7). Meanwhile, $\beta$ cells from $\mathrm{KO}$ recipients expressed genes associated with immune suppression (GABA receptor A $(\mathrm{Gabrg} 3)^{27}, \mathrm{Gad} 2, \mathrm{Cd} 14^{28}$, and $\mathrm{H} 2$ Q7 (Qa-2) ${ }^{14,15}$ (Fig. 5a, b). Compare to WT $\beta$ cells, there were changes in the expression of genes related to improved $\beta$ cell fitness (Slc39a4, Tgfbr2, Fgfr2, Fgfr3, and Aldh1a3) $29-32$ and survival (Gas6, Ptpn13, and Tnfrsf19) ${ }^{33-37}$ (Fig. 5a, b).

To understand the action of Tet 2 in $\beta$ cells, we performed ATAC-seq on the same $\beta$ cell samples analyzed by RNA-seq in Fig. 5a, b. We identified 60,449 open chromatin sites among the five samples profiled. Accessibility at the overwhelming majority of these sites ( $97 \%)$ did not differ substantially between WT and Tet2-KO cells, suggesting that Tet2 deficiency does not lead to widespread chromatin remodeling of $\beta$ cells. About 715 sites $(\sim 1 \%)$ had greater accessibility in KO's (FC >1.5) and $1387(\sim 2 \%)$ had lower accessibility $(\mathrm{FC}<-1.5)$ in Tet2-KO vs WT cells (Fig. 5c). Using HOMER transcription factor (TF) motif enrichment analysis, 97 TF motifs were enriched in peaks with lower accessibility (closing peaks) in KO $\beta$ cells ( $q$ value $<0.05)$ (Supplementary Table 2). Interestingly, motifs for 15 TFs mediating inflammatory responses such as Stats 1, 3, 4, and 5 and Irfs 1, 2, 4, and 8 (Fig. 5d) were specifically enriched in closing peaks, suggesting that inflammatory stress-responsive cisregulatory elements are epigenetically decommissioned in Tet2deficient $\beta$ cells. Fifteen TF motifs were identified as enriched in peaks with higher accessibility (opening peaks) in KO $\beta$ cells ( $q$ value $<0.05)$. Most of these corresponded to TFs controlling islet $\beta$ cell identity and function such as Foxa1, Foxa2, and Rfx and were also enriched in closing peaks in KO $\beta$ cells (Supplementary Table 3), indicating that Tet 2 deficiency results in opening and closing of a subset of islet TF binding sites.

To confirm these findings and the responses of $\beta$ cells to inflammatory mediators, we first screened inducible cytokines and chemokines in islets following IFN $\gamma$ with or without TNFa + IL-1 $\beta$. Il6, Cxcl10, Cxcl16, Ccl2, as well as Tnf were induced by this cytokine cocktail (TNF $\alpha+$ IL- $1 \beta+$ IFN $\gamma$ ) but not Ccl12, Ccl19, or Il1b (Supplementary Fig. 8a).

Previously $\mathrm{Xu}$ et al. described impaired STAT1 signaling in tumor cells cultured with IFN $\gamma^{18}$. Similarly, we identified reduced IFN $\gamma$ response measured by the production of $\mathrm{Cxcl10,}$ Cxcl11 as well as the expression of Pdl1 but not Cxcl9 in KO vs WT islets from B6 mice (Supplementary Fig. 8b). We cultured WT and KO islet cells with TNFa + IL-1 $\beta+$ IFN $\gamma$ and found in Tet2-KO islets reduced induction of Il6, Ccl2, Cxcl10, Cxcl16, and Tnf, which have been linked to $\beta$ cell destructive processes (Fig. 6a). Islets from WT or KO B6 mice were used to avoid any immune cell effects. In addition, we found that the expression of genes associated with $\beta$ cell death such as Fas, Tnfsf10b (TRAIL-R2), and Stat $1^{38,39}$ were also reduced in cultured KO $\beta$ cells (Fig. 6b). DNA from $\beta$ cells were recovered from KO and WT BMT recipients and the enrichment of 5-hydroxymethylcytosine $(5 \mathrm{hmC})$ on the promoter regions $(500 \mathrm{bp}$ within TSS that contains multiple $\mathrm{CpG}$ sites) of genes measured in Fig. 6a, b was compared. Promoter regions of Il-6, Cxcl10, Cxcl16, Fas, and Stat 1 show reduced $5 \mathrm{hmC}$ levels in $\mathrm{KO}$ vs WT $\beta$ cells (Fig. $6 \mathrm{c}$ ). When human islets were cultured with cytokines, we found a similar reduction of chemokine, cytokines as well as death receptors as seen in the mouse experiment when TET2 inhibitor $\mathrm{R}-2 \mathrm{HG}^{40,41}$ was added to the culture (Fig. $6 \mathrm{~d}$ ).

Finally, to determine whether these differences in inflammatory responses are associated with $\beta$ cell survival, we transferred splenocytes from diabetic NOD mice into irradiated WT and KO 

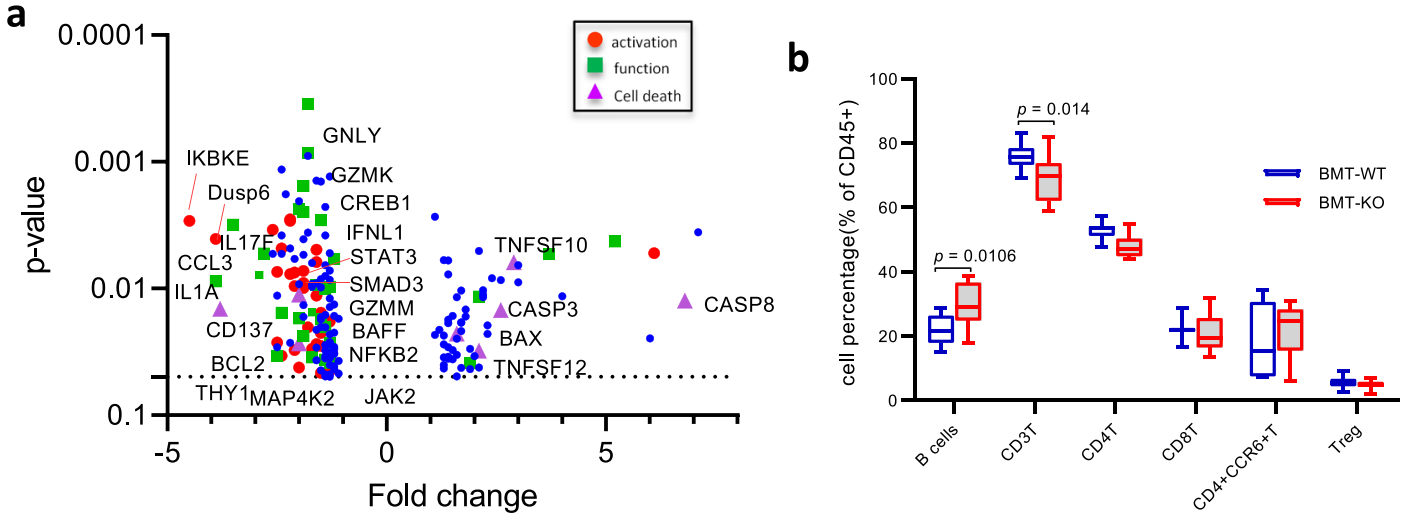

C
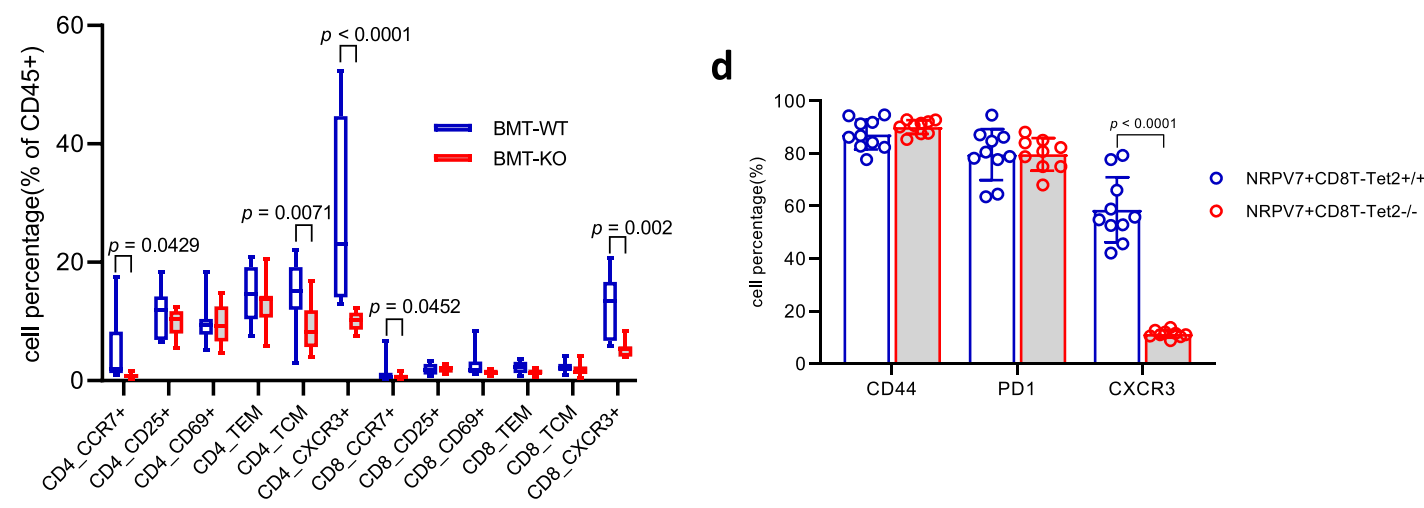

Fig. $4 \mathrm{~T}$ cells from Tet2-KO islets are less activated and show reduced pathogenic phenotypes. a Volcano plot showing transcription differences in islets infiltrates between KO and WT recipients of WT bone marrow. Eight weeks post-BMT, CD45+ islets infiltrates were sorted from KO vs WT recipients and used subsequently for transcription analysis with NanoString Pan-Immunology panel. The data were analyzed with nSolver4.0 analysis software. 189 of 770 genes that were found to be different between infiltrates from WT and KO recipients are shown (after FDR correction, $p<0.05$ ). Genes that are associated with T cell activation, cell function as well as cell death are highlighted with different colors among the 189 genes. Data were from six KO vs six WT samples that were pooled from nine KO and eight WT mice, respectively. b, c FACS analysis of CD45+ cells from pancreatic lymph nodes (pLNs) from KO or WT BMT recipients. The \% of CD45+ cells is shown. (Treg $=$ CD4 + CD25 + CD127lo, TCM $=C D 44+C D 62 L^{\text {hi }}, T E M=C D 44+C D 62 L^{10}$ ). Data were from four-five experiments with pLNs from individual mice. The box indicates $25-75 \%$. The whiskers show the minima to the maxima values and the central line indicates the median. Statistical analysis was performed using two-way ANOVA with Sidak's multiple comparisons test (WT: $n=11$ in (b), 12 in (c); KO: $n=14$ in $\mathbf{b}, 11$ in c). d FACS analysis of the activation status of NRPV7 tetramer-positive IGRP-reactive CD8+ T cells from pLNs of KO and WT BMT recipients. Data were from three independent experiments and each data point represents an individual mouse. Statistical analysis was performed using two-way ANOVA with Sidak's multiple comparisons test.

Table 1 Diseases and bio functions identified by Ingenuity Pathway Analysis of Nanostring data.

\section{Diseases and disorders}

Inflammatory response

Immunological disease

\section{Molecular and cellular functions}

Cellular-to-cell signaling and interaction

Cellular function and maintenance

Cellular development

Cellular growth and proliferation

Cellular movement

Physiological system development and function

Immune cell trafficking

Lymphoid tissue structure and development

\section{Top canonical pathways}

Th1 and Th2 activation pathway

Th1 pathway

p value range
$1.22 \mathrm{E}-04-9.69 \mathrm{E}-49$
$8.60 \mathrm{E}-05-2.59 \mathrm{E}-26$
$p$ value range
$1.22 \mathrm{E}-04-9.69 \mathrm{E}-49$
$1.22 \mathrm{E}-04-1.43 \mathrm{E}-45$
$1.22 \mathrm{E}-04-1.58 \mathrm{E}-42$
$1.22 \mathrm{E}-04-1.58 \mathrm{E}-42$
$1.22 \mathrm{E}-04-8.71 \mathrm{E}-41$
$p$ value range
$1.22 \mathrm{E}-04-9.69 \mathrm{E}-49$
$1.22 \mathrm{E}-04-1.58 \mathrm{E}-42$
$p$ value
$9.14 \mathrm{E}-21$
$8.92 \mathrm{E}-19$

\#Molecules

91

44

\#Molecules

86

85

82

84

62

\#Molecules

90

92

Overlap

15.2\% 23/151

$18.1 \%$ 19/105

Diseases and bio functions identified by Ingenuity Pathway Analysis of PanCancer Immune Profiling Data. CD45 + infiltrates were sorted from KO vs WT recipients 8 weeks post-BMT from WT NOD BM donors ( $n=13-16 \mathrm{KO}$ or WT mice over six separate sortings). About 9000-45,000 cells were lysed for each reaction. Results were summarized into different disease and bio functions (marked in bold). The name of the specific pathway was listed as well as the $P$ value and number of molecules involved in each pathway. 
a

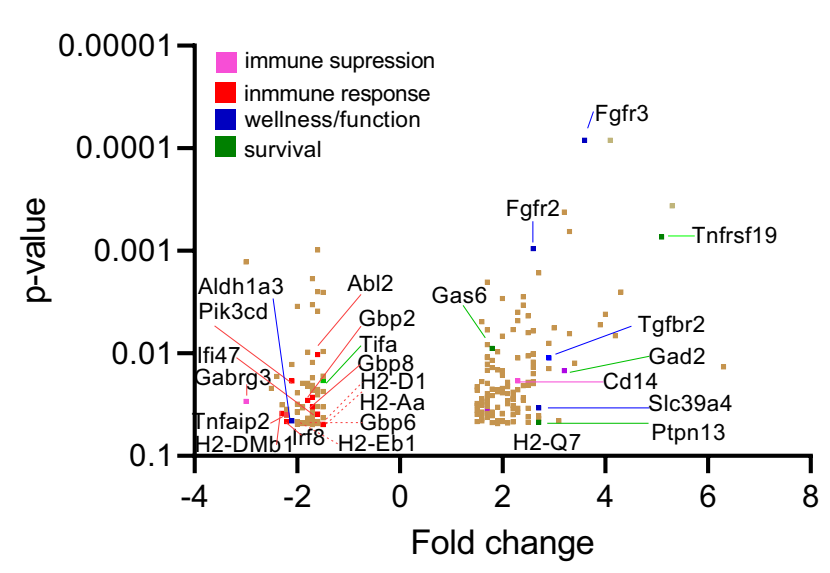

C

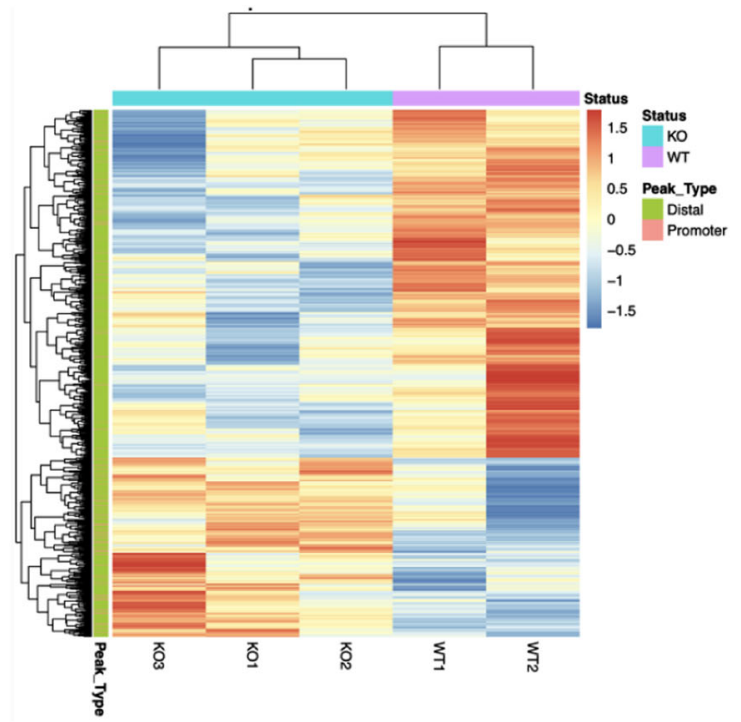

b

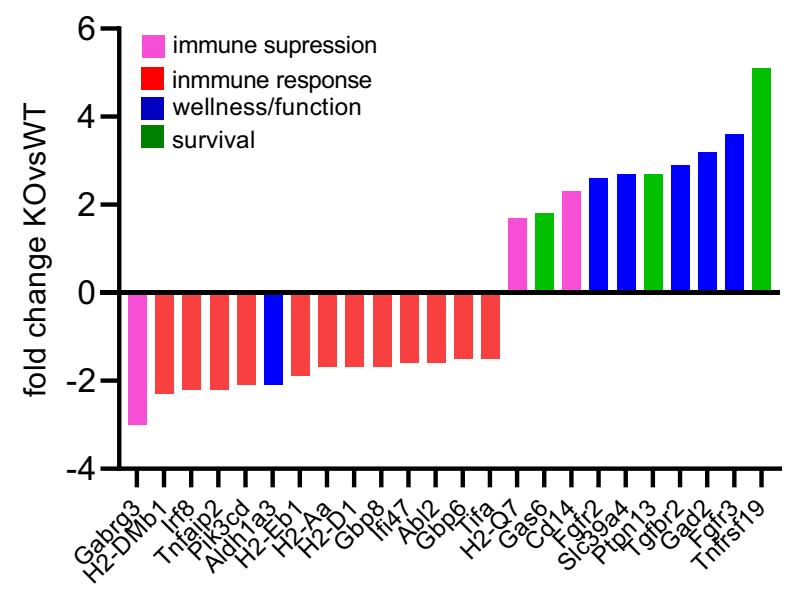

d

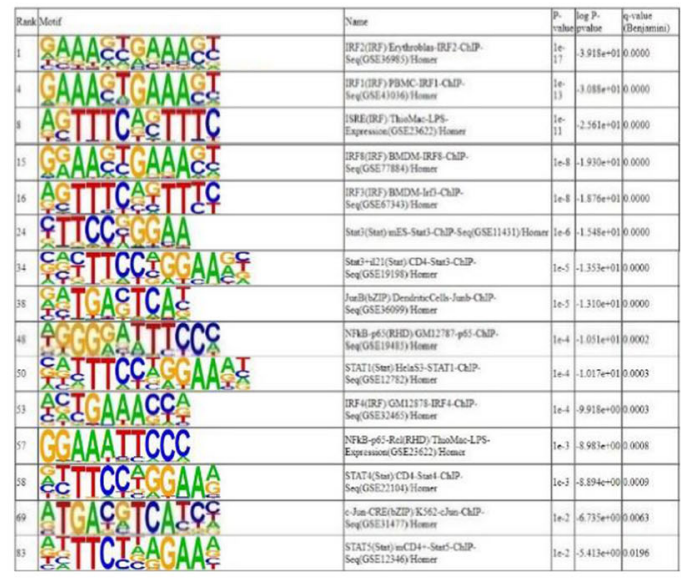

Fig. $5 \beta$ cells lacking Tet2 have reduced inflammatory responses. a Volcano plot showing gene expression differences in $\beta$ cells from KO vs WT recipients of WT bone marrow. Eight weeks after the bone marrow transfer, $\beta$ cells that are TMRE + Zinc + were sorted from KO vs WT recipients and subjected to the cDNA library preparation and bulk RNA-seq analysis. There are 333 differentially expressed genes in total with $p<0.05$ after FDR correction, and 199 with fold change $>1.5$ that are shown in $\mathbf{a}$. Genes of particular interest are color-coded in $\mathbf{a}$ and $\mathbf{b}$ according to their function. c Comparison of ATAC-seq profiles identify chromatin accessibility changes in $\beta$ cells from KO vs WT recipients of WT bone marrow. Of 60,449 accessible sites identified across the five samples, 715 ( 1\%) had increased accessibility (FC >1.5) and $1387(\sim 2 \%)$ had reduced accessibility in (FC $<-1.5)$ in KO's, respectively. d HOMER motif analysis identified 97 TF motifs enriched in peaks with decreased accessibility (closing peaks) in KO $\beta$ cell samples $(q$ value $<0.05), 15$ of which are inflammatory mediators. The rank of the 97 TF motifs was sorted by $q$ value. Data were from three sortings and three-four mice per group per sorting.

recipients and followed them for the development of diabetes. The median time to hyperglycemia was 9.5 weeks in the WT recipients whereas none of the six $\mathrm{KO}$ recipients developed diabetes for more than 20 weeks after adoptive transfer $(p=0.0041)$ (Fig. 6e).

\section{Discussion}

We have shown that TET2 can control immune-mediated destruction of human and murine $\beta$ cells through the interactions with immune cells and $\beta$ cell-intrinsic mechanisms. Tet 2 expression is increased during the progression of diabetes in islet cells and in $\beta$ cells. However, a subpopulation of $\beta$ cells that we previously showed was protected from autoimmune killing, has lower levels of Tet 2 expression. In human pancreases, $\beta$ cells also express TET2 under inflammatory conditions, but islets from normal individuals and from patients with long-standing T1D have $\beta$ cells without detectable TET2 expression. Our findings indicate that TET2 is associated with, but not sufficient for, killing of $\beta$ cells, since we also found increased TET2 expression in $\beta$ cells from patients with autoimmune pancreatitis who do not develop diabetes. Instead, our studies in Tet2-KO mice indicate that in the absence of Tet2, $\beta$ cells are protected from immune-mediated killing. We used three complementary model systems: BM transplantation, islet transplantation, and adoptive transfer of diabetogenic splenocytes to identify the interactive relationships between Tet 2 expression in $\beta$ cells and immune cells and show that Tet2-KO islets are resistant to killing even in the presence or transfer of diabetogenic T cells. The Tet2-KO $\beta$ cells show transcriptional differences, compared to WT in responses to inflammatory cytokines in genes associated with $\beta$ cell wellness, 

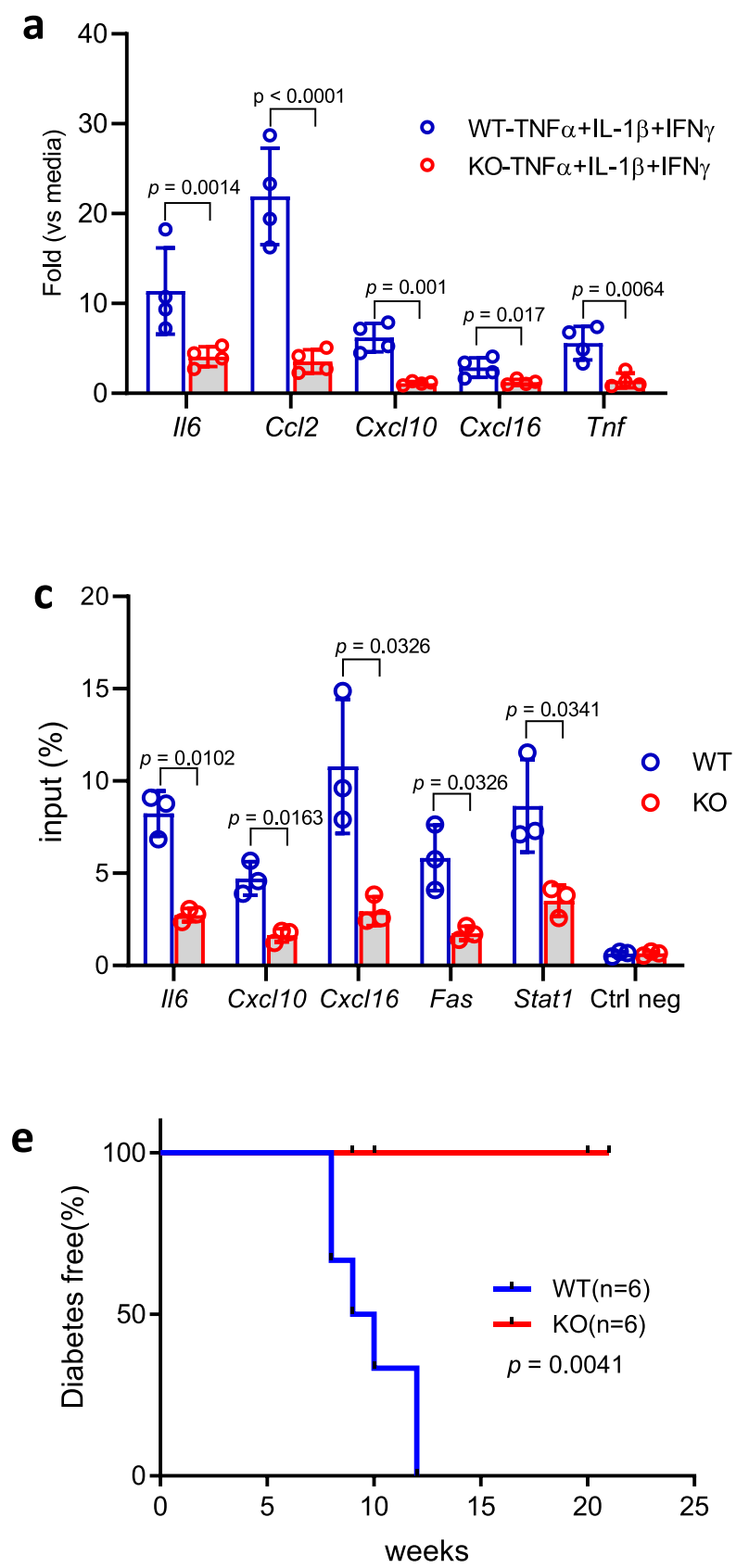

b
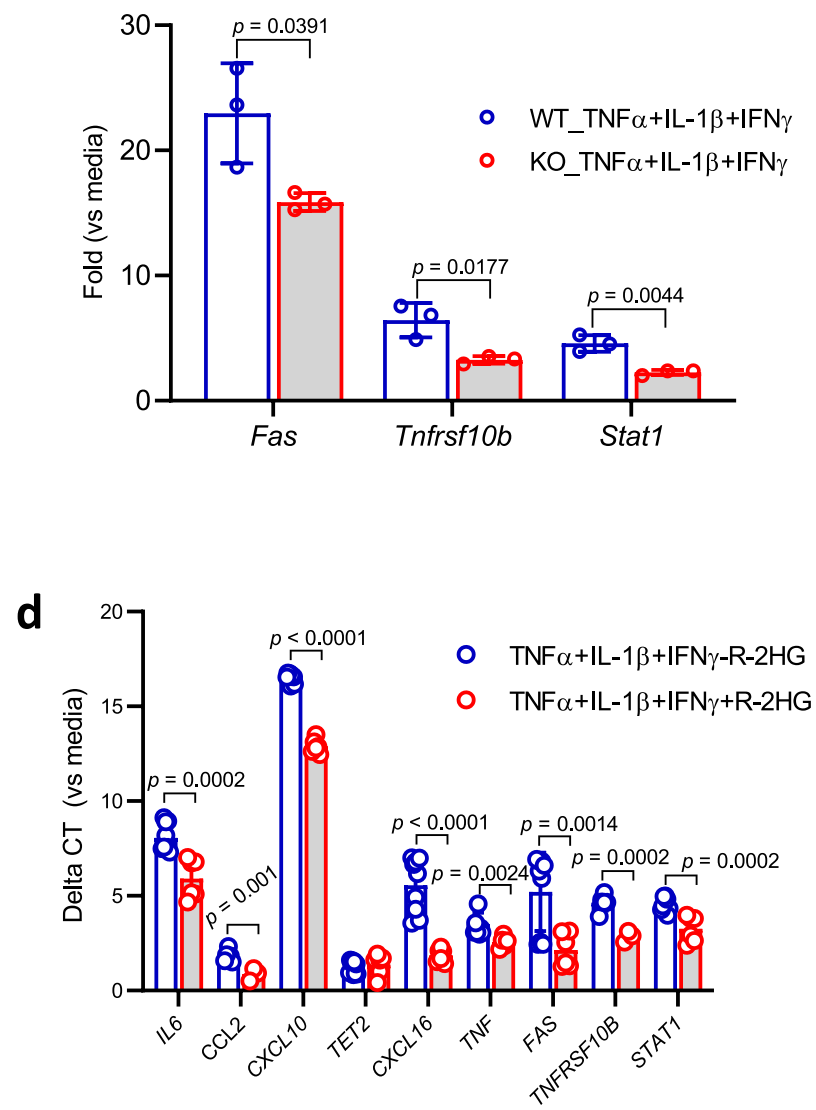

Fig. 6 Tet2-KO B cells show reduced inflammation response in vitro and are resistant to killing by diabetogenic cells in vivo. Transcription analysis of cytokines and chemokines (a) as well as cell death receptors (b) in KO vs WT islets from B6 mice following culture with the indicated cytokines for $24 \mathrm{~h}$. Data were mean \pm SD of three-four independent experiments, four mice per group per experiment. Statistical analysis was performed using a two-tailed unpaired $t$-test without correction for multiple comparisons. c Enrichment of $5 \mathrm{hmC}$ at the promoter regions of indicated genes was examined by hMeDIPqPCR. Comparisons were made between $\beta$ cells from KO vs WT NOD recipients of WT NOD bone marrow. Data were mean \pm SD of three independent experiments, three-five mice per group per experiment. Statistical analysis was performed using a two-tailed unpaired t-test without correction for multiple comparisons. $\mathbf{d}$ Transcription analysis of the genes studied in (a and $\mathbf{b}$ ) in human islets after culture with TNF $\alpha+$ IL-1 $\beta+$ IFN $\gamma$ with or without TET inhibitor $\mathrm{R}-2 \mathrm{HG}$ for $24 \mathrm{~h}$. The $\mathrm{Ct}$ of cytokine group with or without R-2HG was compared to islets in media supplemented with or without R-2HG, respectively. Data (mean \pm SD) were from three experiments, each with 4000-8000 islet equivalency (IEQ) from each nondiabetic donor. Statistical analysis was performed using a two-tailed unpaired $t$-test without correction for multiple comparisons. e Diabetes incidence in Tet2-WT or KO NOD mice that received by adoptive transfer $1.5 \times 10^{7}$ diabetogenic splenocytes from diabetic WT NOD mice after half lethal dose irradiation ( 650 rads). Mice were followed up to 21 weeks post adoptive transfer. Statistical analysis was performed using Log-rank curve comparison. $n=6$ for each group.

function, survival, and immune suppression, and reduced expression of genes associated with immune responses and activation. Importantly, there was also reduced chromatin accessibility at putative regulatory elements containing binding motifs of inflammatory mediators in Tet2-KO $\beta$ cells. These molecular changes were confirmed by functional studies that showed reduced secretion of cytokines and chemokines when the Tet2$\mathrm{KO}$ islets were cultured with inflammatory cytokines. As a result, Tet2-KO islets fail to activate immune responses and autoreactive $\mathrm{T}$ cells, resulting in reduced autoimmune elimination of Tet2-KO $B$ cells compared to WT. In summary, our studies have identified a mechanism that controls interactions between $\beta$ cells and 
immune cells that leads to immune-mediated killing and suggest that inhibiting this pathway may lead to improved survival of $\beta$ cells in the setting of autoimmunity.

In human pancreatic sections, we found differences in TET2 expression within islet cells and between pancreases from individuals with different stages of $\beta$ cell destruction. We found expression in both the nuclear and cytoplasm, consistent with reports in neurons ${ }^{42}$. In patients with long-standing $\mathrm{T} 1 \mathrm{D}$, the absence of TET2 expression suggests an association with $\beta$ cell survival or possibly $\beta$ cells that do not respond to inflammatory mediators.

Our studies of the control of $\beta$ cell responses by Tet 2 provide insight into the mechanisms that account for these histologic findings. A fully pathologic autoimmune repertoire developed in the Tet2-KO recipients of WT BM. However, these cells did not receive the same activation signals and did not cause $\beta$ cell killing as shown by our analysis of their transcriptome, when compared to islet infiltrates from the WT BMT recipients. The absence of TET2 expression on $\beta$ cells, therefore, could potentially explain the ability of some $\beta$ cells to survive even when autoreactive $\mathrm{T}$ cells remain in the host. The Tet2-KO BM recipients developed diabetogenic $\mathrm{T}$ cells since splenocytes were able to transfer diabetes to NOD/scid recipients at a rate similar to WT cell donors. Consistent with these findings, islets from the Tet2-KO mice showed significantly delayed rejection compare to islets from WT donors when they were transplanted into WT NOD recipients. Even in the same mouse, the WT but not KO islets were preferentially destroyed.

Tet 2 deficiency may affect the survival of $\beta$ cells through direct or indirect pathways. ATAC-seq revealed reduced chromatin accessibility in Tet2-deficient $B$ cells at putative binding sites for Stat and Irf TFs that have been associated with cytokine signaling. Gene expression may occur from local epigenetic modifications and therefore decreased expression is expected with Tet2 deficiency ${ }^{43}$. Previous studies of Tet2-deficient tumor cells identified impaired STAT1 signaling that prevented the expression of chemokines and PD-L1 expression and enabled tumors to evade anti-PD-L1 immune therapy ${ }^{18}$. These investigators described a disrupted IFN $\gamma$-JAK-STAT signaling cascade in Tet2-deficient tumor cells that we also found with $\beta$ cells.

These observations indicate that the islet cells themselves deliver signals to the autoreactive immune cells that leads to their activation and $\beta$ cell killing. These signals most likely are the result of differences in the Tet $2-\mathrm{KO} \beta$ cells since the antigenpresenting cells in the transplant recipients were from the WT BM donor. Our data, in Supplementary Fig 8, show that IFN $\gamma$ inducible chemokines (CXCL10 and CXCL9), as well as PD-L1, are reduced in the $\mathrm{KO} \beta$ cells, consistent with the inaccessibility of STAT1 signaling (Fig. 5d). Groom et al. described a CXCR3 inflammatory loop in which the IFN $\gamma$-induced chemokines recruit CXCR3-expressing $\mathrm{T}$ cells to inflammatory sites ${ }^{44}$. The production of IFN $\gamma$ by the CXCR3 $+\mathrm{T}$ cells can provide a "feed forward" in which additional CXCR3-expressing inflammatory cells, such as those thought to mediate autoimmune diabetes are recruited. Thus, the inability to respond to IFN $\gamma$ may block this loop and result in the reduced frequency of the pathologic $\mathrm{T}$ cells ${ }^{23,45-48}$. A unique feature of this mechanism of protection is that it does not dependent on the specificity or the ability of the immune cells to respond to the target $\beta$ cells per se but rather on the ability of the $\beta$ cells to respond to inflammatory mediators and recruit and activate the $\mathrm{T}$ cells. In addition, as a result of the loss of Tet2, islet cells express reduced levels of molecules that are directly responsible for killing including Fas and Tnfrsf10b.

In addition to the participation of the $\beta$ cells in immune cell interactions, we also identified intrinsic pathways to account for enhanced $\beta$ cell survival in Tet2-KO $\beta$ cells. We found improved survival when $\beta$ cells were cultured with inflammatory cytokines as well as infiltrating immune cells, both of which may mediate $\beta$ cell killing. In the Tet2-KO $\beta$ cells, expression of GABA receptor A (Gabrg3), which is associated with immune suppression, was reduced and there was increased expression of Tnfrsf19, Fgfr2, and Fgfr3. Increased Tnfrsf 19 expression was shown to attenuate TNF $\beta$ R signaling and improve $\beta$ cell survival ${ }^{29,30}$. Fgfr2 and Fgfr3 are growth factor receptors, and attenuation of FGF signaling by expressing dominant-negative forms of the FGFRs receptors in mouse $\beta$ cells leads to diabetes ${ }^{31}$. Gas6, which is linked to $\beta$ cell proliferation, was also increased in the expression on the $\mathrm{KO} \beta$ cells ${ }^{49}$. These cell-intrinsic mechanisms may account for the resistance of the cells to killing by inflammatory cytokines in the absence of extrinsic cells.

There are a number of limitations of our studies. First, TET2 loss of function mutations occurs frequently in humans ${ }^{18}$. Therefore, the applicability of our findings in the inbred NOD mouse needs further confirmation. Moreover, some have questioned the model's relevance to human disease since there are a number of nonspecific interventions that may modulate spontaneous diabetes in this model ${ }^{50}$. Tet 2 causes demethylation of methylcytosines, and therefore the relationship between the loss of this enzymatic activity and decreased transcription of genes is somewhat paradoxical. However, our previous studies showing increased activity of Dnmts suggest a mechanism that, if unopposed, could lead to these outcomes ${ }^{51}$. In our Tet2-deficient NOD mice and recipients of bone marrow transplants (BMTs), Tet2 was absent throughout the development of immune cells. We do not know if there is a critical time(s) when Tet2 expression is involved in $\beta$ cell killing as well as immune activation but our adoptive transfer studies with diabetogenic T cells would suggest that absence of Tet 2 can be protective even when $\beta$ cells are attacked by fully matured effector cells. In addition, the relationship, particularly in human tissues, between the autoreactive repertoire, TET2 expression, and $\beta$ cell killing will require further studies with human $\beta$ and effector T cells. Lastly, there may be an effect of other environmental factors, on the activation of $\mathrm{T}$ cells in the Tet2-deficient mice, such as the microbiome that may affect their activation ${ }^{52}$.

In summary, our studies have identified an interaction between $\beta$ cells and immune cells that regulates immune cell activation, recruitment, and killing. Expression of Tet 2 is required for $\beta$ cells to produce chemokines and cytokines that are needed for the activation and pathologic function of diabetogenic T cells. In its absence, $\beta$ cells resist cell- or cytokine-mediated killing even when potentially autoreactive cells are present through cell-intrinsic mechanisms as well as avoiding activation of immune cells. These studies suggest a potential future strategy to protect insulinproducing cells from immune-mediated killing in the setting of autoimmunity or even for engineering more resilient and/or resistant $\beta$ cell replacements to avoid recurrent immune elimination.

\section{Methods}

Mice. C57BL/6 $6^{\text {Tet2-1- }}$ male breeder under B6 background was purchased from The Jackson lab and backcrossed with NOD females for over 14 generations. Female NOD, B6, and NOD/scid mice were also obtained from The Jackson Laboratory and maintained under pathogen-free conditions. All protocols were approved by the Yale Institutional Animal Care and Use Committee. Animals were housed under the following facility conditions: Temperatures: $21-23^{\circ} \mathrm{C}$; Humidity: 50-60\%. Lighting: 12:12 (i.e., $12 \mathrm{~h}$ of light from 7 a.m. -7 p.m. then $12 \mathrm{~h}$ of dark from 7 p.m.-7 a.m.).

Intraperitoneal glucose tolerance test (IPGTT). B6 Tet2-WT, HET, and KO mice fasted for $16 \mathrm{~h}$. Blood glucose levels were measured 0, 15, 30, 60 and $120 \mathrm{~min}$ after glucose injection ( $2 \mathrm{~g} / \mathrm{kg}$ body weight). IPGTT result was presented as the area under the curve using trapezoidal rule ${ }^{53}$ and then divide by the total assay time (120 min). 
Human islets and pancreas tissues. Human islet samples were obtained from adult, nondiabetic organ donors from the Prodo Labs (details listed in Supplementary Table 1). Pancreas tissues from donors with autoimmune pancreatitis $(n=5)$ or chronic pancreatitis $(n=3)$ were obtained from the Department of Pathology at Yale and histologic slides of the pancreas were obtained from normal individuals $(n=8)$, nondiabetic donors who were autoantibody $+(n=7)$, and Cpeptide + patients with T1D of relatively short duration $(n=7)$ from nPOD. The use of human tissues and islets was considered "not human subjects research" as per institutional policies (Yale Institutional Review Board). It did not involve data obtained through intervention or interaction with the individual and did not contain identifiable private information.

\section{Immunofluorescent labeling of human pancreas. To identify TET2 protein} expression in pancreatic islet cells, formalin-fixed, paraffin-embedded sections (5$\mu \mathrm{m}$ thickness) of human pancreas were incubated with primary antibodies against TET2 (Abcam, cat\# 230358), insulin (DAKO, cat\# A0564), and CD45 (DAKO, clone\# $2 \mathrm{~B} 11+\mathrm{PD} 7 / 26)$ overnight at $4{ }^{\circ} \mathrm{C}$, washed and processed with the appro priate secondary fluorescent-conjugated antibodies. Subsequently, sections were dyed with $0.7 \%$ Sudan Black and $\mathrm{CuSO} 4$ to quench auto-fluorescence and counterstained with DAPI ${ }^{54}$. Images were acquired on an UltraVIEW VoX (PerkinElmer) spinning disc confocal Nikon Ti-E Eclipse microscope using the Volocity 6.3 software (Improvision). Image J was used to quantify the fluorescence intensity of TET2 staining in the islets in different clinical conditions. Twenty islets were picked per condition was from three-seven donors.

Mouse islet isolation and $\boldsymbol{\beta}$ cell staining and purification. Mouse islets were handpicked with a stereomicroscope after collagenase digestion of pancreases and single-cell suspensions were prepared ${ }^{15,17}$. For $\beta$ cell enrichment, single islet cells were stained with TMRE and FluoZin-3 and sorted with a FACSAria II (BD). In some experiments, intracellular staining with anti-insulin (R\&D Systems, Clone \# 182410), anti-Tet2 (Cell Signaling Technologies, clone\# D6C7K), and antiglucagon (Abcam, clone\# K79bB10) was used at $2.5 \mathrm{mg} / \mathrm{ml}$ to identify $\beta$ cells or a cells and analyzed or sorted with an LSRFortessa (BD).

Flow cytometry on immune cells. For standard surface staining, lymphocytes $\left(10^{6}\right.$ cells/sample) were washed with PBS and incubated for $30 \mathrm{~min}$ at $4{ }^{\circ} \mathrm{C}$ (dark) in $100 \mu \mathrm{l}$ PBS plus $2 \%$ FBS with indicated fluorochrome-labeled monoclonal antibodies. After washing with PBS two times, 30,000-50,000 live cells were analyzed by flow cytometry. For surface staining, CD45 (clone\# 30-F11, cat\#103125), CD4 (clone\#GK1.5, cat\#100411), CD8 (clone\#53-6.7, cat\#100727), CD25 (clone\#3C7, cat\#101903), CD69 (clone\#H1.2F3, cat\#104505), CXCR3 (clone\#CXCR3-173, cat\#126535), CCR7 (clone\#4B12, cat\#120113), CD44 (clone\#IM7,cat\#103015), and CD62L (clone\#MEL-14, cat\#104407) were diluted to a working concentration of $2 \mathrm{mg} / \mathrm{ml}$. All antibodies were from BioLegend. Gating strategies are included as Supplementary Fig. 9.

Islet cultures. Human or mouse islets were rested upon arrival or isolation in complete RPMI media for $3 \mathrm{~h}$ before aliquoting ( 100 per well) into a non-treated 24-well plate and culture for 18-24 h with or without cytokines. All mouse and human cytokines were used at $10 \mathrm{ng} / \mathrm{ml}$, except for human IL-6 at $20 \mathrm{ng} / \mathrm{ml}$ and human IL-17A at $50 \mathrm{ng} / \mathrm{ml}$. TET inhibitor R-2HG was used at $400 \mathrm{nM}$. The cells were then harvested for RNA extraction and transcriptional analysis of genes of interest. In cytokine or immune cell-mediated killing experiments, mouse islets were dispersed with accutase and cultured as single cells overnight before harvest for live/dead analysis by flow or cell death receptors analysis by qRT-PCR.

Adoptive transfer of diabetogenic splenocytes. Tet2-KO or WT NOD mice ( 8 weeks of age) were irradiated with a split dose $(2 \times 325$ rads, $2-3 \mathrm{~h}$ apart, 650 rads total) and splenocytes $\left(1 \times 10^{7}\right)$ from a WT NOD mouse with recent-onset DM were transferred i.p. within $3 \mathrm{~h}$ of irradiation. The time to diagnosis of diabetes (i.e., glucose $>250 \mathrm{mg} / \mathrm{dl} \times 2$ ) was recorded after the adoptive transfer.

Streptozotocin (STZ) treatment. Eight to 10-week-old Tet2-WT and KO B6 mice were given a single dose of STZ $(200 \mathrm{mg} / \mathrm{kg}$, i.p) and followed daily following the treatment.

Bone marrow transplant (BMT). For BMTs, donor WT NOD mice 6-8 weeks of age were euthanized by $\mathrm{CO}_{2}$. BM was flushed with ice-cold HBSS and the erythrocytes were removed by lysis. The recipient KO or WT mice (4-8 weeks of age) were irradiated with split dose $(2 \times 550$ rads, $2-3 \mathrm{~h}$ apart, 1100 rads total $)$ and donor BM $\left(10 \times 10^{6}\right.$ cells $)$ transferred i.v. within $3 \mathrm{~h}$ of irradiation ${ }^{55}$. Recipients were followed for hyperglycemia (glucose $>250 \mathrm{mg} / \mathrm{dl} \times 2$ ) and diabetic incidence was recorded at the time after BMT.

Islet transplant. About 250-300 handpicked islets were pooled from 5-6 weeks KO or WT donors (two-three donors per recipient). They were transplanted under the kidney capsule of WT NOD recipients (6 weeks of age). Two weeks later, diabetogenic splenocytes were given i.p.to the transplant recipients $\left(1 \times 10^{7}\right.$ cells per recipient). Blood glucose was measured twice weekly and diabetes incidence was followed and recorded at the age of islets recipients.

Low-input RNA-seq and data analysis. Viable $\beta$ cells were enriched by FACS sorting from $\mathrm{KO}$ or WT recipients 8 weeks post-BMT from WT BM donors. RNA was extracted and reverse transcription and cDNA amplification were performed using 10 ng of RNA using the SMARTer Ultra Low RNA kit (Clontech Laboratories) as previously reported ${ }^{15}$. Sequencing libraries were prepared using the Nextera XT DNA Sample Preparation kit (Illumina). Libraries from eight samples were pooled and sequenced with a $2 \times 100 \mathrm{bp}$ paired-end protocol on the HiSeq 4000 Sequencing System (Illumina), occupying half a lane with 300 million reads. The sequencing was done at the Yale Stem Cell Center Genomics Core. The reads were mapped to mouse reference transcriptome $\mathrm{mm} 9$ and mRNA was quantified. RNA-Seq data were analyzed with Partek Flow software (v6.6).

NanoString Pan-Immunology Panel Analysis of islet infiltrates. Analysis was done with the NanoString Technologies Pan-Immunology panel as per the manufacturer's instructions. Briefly, 9000-45,000 CD45+ islet infiltrates were sorted from KO or WT BMT recipients and cell pellets were lysed in 33\% RLT lysis buffer (QIAGEN). Up to $3.5 \mu$ lysate was used directly in a $15 \mu$ l hybridization reaction which was kept at $65^{\circ} \mathrm{C}$ for $24 \mathrm{~h}$. The hybridization product was prepped and used with the digital analyzer nCounter MAX/FLEX system. The data were analyzed with nSolver4.0 analysis software (NanoString Technologies). Differentially expressed genes ( $P$ value $<0.05$ after FDR correction) were identified and pathways were analyzed with IPA and upstream regulator analysis (https:// www.qiagenbioinformatics.com/products/ingenuity-pathway-analysis/).

RNA extraction and RT-PCR analysis. RNA was extracted with RNeasy Plus Mini Kit (QIAGEN, cat\#74134), and the High-Capacity cDNA Reverse Transcription Kit was used for cDNA synthesis (Applied Biosystems, cat\#4368814). Primer pairs were used in this study together with a QuantiFast SYBR Green PCR kit (QIAGEN). The sequences of primer pairs used in this study are listed in Supplementary Table 4. The Actb housekeeping gene was used to normalize the input RNA in all real-time qPCR assays. Gene expression is presented as Delta $C t=(C t$ of $A c t b-C t$ of target gene) +20 or in other cases as relative fold to control. In Supplementary Fig. 1e, RNA was recovered from perm/fixed cells with RecoverAll ${ }^{\text {TM }}$ Total Nucleic Acid Isolation Kit for FFPE (Invitrogen, AM1975). Taqman probes were used in $\mathrm{qPCR}^{15}$.

ATAC-seq and data analysis. ATAC-seq libraries were prepared from FACS sorted mouse islet $\beta$ cells ${ }^{56}$. After wash and lysis, the cell pellet was resuspended in $50 \mathrm{ul}$ of transposition mixture (Illumina Tagment DNA TDE1 Enzyme and Buffer) and incubated for $30 \mathrm{~min}$ at $37^{\circ} \mathrm{C}$. Transposed DNA was cleaned with a Qiagen MinElute kit and stored frozen at $-20^{\circ} \mathrm{C}$ until indexing as decribed ${ }^{57}$. Libraries were sequenced on an Illumina NovaSeq S1 with $2 \times 101$ bp cycles. Raw sequence reads were quality trimmed using Trimmomatic version $0.32^{58}$ with the following parameters “TRAILING:3 SLIDINGWINDOW:4:15 MINLEN:36." Trimmed reads were aligned to the mouse genome ( $\mathrm{mm} 10)$ using BWA version $0.7 .12^{59}$, specifically using the bwa mem -M option. Duplicate reads were marked and removed using "MarkDuplicates" from Picard-tools version 1.95 (http://

broadinstitute.github.io/picard/). The quality of aligned reads was examined using Ataqv v.1.0.0 ${ }^{60}$. After preprocessing and quality filtering, peaks were called on alignments with MACS version 2.1.0.20151222 ${ }^{61}$ using the parameters "-p 0.0001 -g mm -f BAMPE -nomodel -nolambda -B -keep-dup all -call-summits”. Peaks located in blacklisted regions of the genome were removed. Overlapping peaks from all samples were merged with BEDTools version 2.26.0 62 resulting in 60,449 consensus peak regions. Raw read counts in these peaks for each sample were determined using the R package DiffBind_2.4.8 ${ }^{63}$. To identify peaks with differing chromatin accessibility between WT and KO samples, the log mean normalized reads (log2 counts per million) were compared between the two sample groups. Peaks with a differing accessibility $>1.5$-fold in either group were used for TF motif enrichment analysis. The HOMER suite version $4.6^{64}$ and "findMotifsGenome.pl" script with parameters "mm10 -size 200" was used to determine TF motifs enriched ( $q$ value $<0.05)$ in the peaks of interest.

Hydroxymethylated DNA immunoprecipitation and qPCR. The experiments were carried out using the hMeDIP kit (Active Motif, 55010) according to the manufacturer's instructions. Briefly, $\beta$ cells were enriched from islets 8 weeks after BMT for genomic DNA recovery with the DNeasy Blood \& Tissue Kit (QIAGEN, 69504), followed by digestion using restrictive enzyme Mse I (NEB, R0525S). Digested DNA was purified through with DNA purification column (Qiagen, 28104). Purified DNA ( $1 \mu \mathrm{g})$ was immunoprecipitated with $2.5 \mu \mathrm{g}$ of mouse anti$5 \mathrm{hmC}$ antibody from the kit and was incubated overnight at $4{ }^{\circ} \mathrm{C}$. The magnetic beads were added to the DNA-antibody mixture for $2 \mathrm{~h}$ incubation at $4{ }^{\circ} \mathrm{C}$ and isolation of immunoprecipitated DNA was performed according to the kit instructions. SYBR ${ }^{\circledast}$ Green Supermix (Bio-Rad) on a Bio-Rad CFX96 Real-Time system was used to perform qPCR, as indicated by the manufacturer's protocol. Eluted DNA was undergone qPCR as template and primers adjacent (500 bp to the 
transcription starting site (TSS)) to the promoter regions of target genes was used for amplification to analyze the changes in the local $5 \mathrm{hmC}$ signal in the respective promoter regions. The Ct values of each sample were used in the post-PCR data analysis. The relative enrichments (after normalization against control IgG) of the indicated DNA regions were calculated using the Percent Input (10\% of the total DNA used in the reaction) Method according to the manufacturer's instructions.

Statistical analysis. The number of samples per group and experiment repeats, as well as the statistical tests used, is indicated in each figure legend.

Reporting Summary. Further information on research design is available in the Nature Research Reporting Summary linked to this article.

\section{Data availability}

The ATAC-seq and RNA-seq data that support the findings of this study have been deposited in NCBI BioProject (https://www.ncbinlm.nih.gov/bioproject/?term=PRJNA630597) with the accession code PRJNA630597. All other data are available from the corresponding author upon reasonable request. Source data are provided with this paper.

Received: 31 May 2020; Accepted: 6 August 2021;

Published online: 20 August 2021

\section{References}

1. Keenan, H. A. et al. Residual insulin production and pancreatic ss-cell turnover after 50 years of diabetes: Joslin Medalist Study. Diabetes 59, 2846-2853 (2010).

2. Neyman, A. et al. Persistent elevations in circulating INS DNA among subjects with longstanding type 1 diabetes. Diabetes Obes. Metab. 21, 95-102 (2019).

3. Soleimanpour, S. A. \& Stoffers, D. A. The pancreatic beta cell and type 1 diabetes: innocent bystander or active participant? Trends Endocrinol. Metab. 24, 324-331 (2013).

4. Cooper, J. D. et al. Confirmation of novel type 1 diabetes risk loci in families. Diabetologia 55, 996-1000 (2012).

5. Eizirik, D. L. et al. The human pancreatic islet transcriptome: expression of candidate genes for type 1 diabetes and the impact of pro-inflammatory cytokines. PLoS Genet. 8, e1002552 (2012).

6. Kutlu, B. et al. Detailed transcriptome atlas of the pancreatic beta cell. BMC Med. Genomics 2, 3 (2009).

7. Larger, E., Becourt, C., Bach, J. F. \& Boitard, C. Pancreatic islet beta cells drive $\mathrm{T}$ cell-immune responses in the nonobese diabetic mouse model. J. Exp. Med. 181, 1635-1642 (1995)

8. Itoh, A. \& Maki, T. Protection of nonobese diabetic mice from autoimmune diabetes by reduction of islet mass before insulitis. Proc. Natl Acad. Sci. USA 93, 11053-11056 (1996).

9. Liston, A., Todd, J. A. \& Lagou, V. Beta-cell fragility as a common underlying risk factor in type 1 and type 2 diabetes. Trends Mol. Med. 23, 181-194 (2017).

10. Cnop, M. et al. Mechanisms of pancreatic beta-cell death in type 1 and type 2 diabetes: many differences, few similarities. Diabetes 54, S97-S107 (2005).

11. Gagnerault, M. C., Luan, J. J., Lotton, C. \& Lepault, F. Pancreatic lymph nodes are required for priming of beta cell reactive T cells in NOD mice. J. Exp. Med. 196, 369-377 (2002).

12. Hoglund, P. et al. Initiation of autoimmune diabetes by developmentally regulated presentation of islet cell antigens in the pancreatic lymph nodes. $J$. Exp. Med. 189, 331-339 (1999).

13. Thompson, P. J. et al. Targeted elimination of senescent beta cells prevents type 1 diabetes. Cell Metab. https://doi.org/10.1016/j.cmet.2019.01.021 (2019).

14. Lee, $\mathrm{H}$. et al. Beta cell dedifferentiation induced by IRElalpha deletion prevents type 1 diabetes. Cell Metab. 31, 822-836 e825 (2020).

15. Rui, J. et al. beta cells that resist immunological attack develop during progression of autoimmune diabetes in NOD mice. Cell Metab. https:// doi.org/10.1016/j.cmet.2017.01.005 (2017).

16. Stefan-Lifshitz, M. et al. Epigenetic modulation of beta cells by interferonalpha via PNPT1/mir-26a/TET2 triggers autoimmune diabetes. JCI Insight https://doi.org/10.1172/jci.insight.126663 (2019).

17. Rui, J. et al. Methylation of insulin DNA in response to proinflammatory cytokines during the progression of autoimmune diabetes in NOD mice. Diabetologia 59, 1021-1029 (2016).

18. $\mathrm{Xu}, \mathrm{Y}$. P. et al. Tumor suppressor TET2 promotes cancer immunity and immunotherapy efficacy. J. Clin. Investig. 130, 4316-4331 (2019).

19. Cimmino, L. et al. Restoration of TET2 function blocks aberrant self-renewal and leukemia progression. Cell 170, 1079-1095 e1020 (2017).

20. Carrillo-Jimenez, A. et al. TET2 regulates the neuroinflammatory response in microglia. Cell Rep. 29, 697-713 e698 (2019).
21. Ichiyama, K. et al. The methylcytosine dioxygenase Tet2 promotes DNA demethylation and activation of cytokine gene expression in T cells. Immunity 42, 613-626 (2015).

22. Cai, Z. et al. Inhibition of inflammatory signaling in Tet 2 mutant preleukemic cells mitigates stress-induced abnormalities and clonal hematopoiesis. Cell Stem Cell 23, 833-849 e835 (2018).

23. Pirot, P., Cardozo, A. K. \& Eizirik, D. L. Mediators and mechanisms of pancreatic beta-cell death in type 1 diabetes. Arq. Bras. Endocrinol. Metab. 52, 156-165 (2008).

24. Vella, P. et al. Tet proteins connect the O-linked N-acetylglucosamine transferase Ogt to chromatin in embryonic stem cells. Mol. Cell 49, 645-656 (2013).

25. Stiles, B. L. et al. Selective deletion of Pten in pancreatic beta cells leads to increased islet mass and resistance to STZ-induced diabetes. Mol. Cell. Biol. 26, 2772-2781 (2006).

26. Wang, L. et al. Deletion of Pten in pancreatic ss-cells protects against deficient ss-cell mass and function in mouse models of type 2. Diabetes Diabetes 59, 3117-3126 (2010)

27. Bhandage, A. K. et al. GABA regulates release of inflammatory cytokines from peripheral blood mononuclear cells and $\mathrm{CD} 4(+) \mathrm{T}$ cells and is immunosuppressive in type 1 diabetes. EBioMedicine 30, 283-294 (2018).

28. Osterbye, T. et al. A subset of human pancreatic beta cells express functional CD14 receptors: a signaling pathway for beta cell-related glycolipids, sulfatide and beta-galactosylceramide. Diabetes/Metab. Res. Rev. 26, 656-667 (2010).

29. Hardy, A. B. et al. Zip4 mediated zinc influx stimulates insulin secretion in pancreatic beta cells. PloS ONE 10, e0119136 (2015)

30. Nomura, M. et al. SMAD2 disruption in mouse pancreatic beta cells leads to islet hyperplasia and impaired insulin secretion due to the attenuation of ATP-sensitive K+ channel activity. Diabetologia 57, 157-166 (2014).

31. Hart, A. W., Baeza, N., Apelqvist, A. \& Edlund, H. Attenuation of FGF signalling in mouse beta-cells leads to diabetes. Nature 408, 864-868 (2000).

32. Kim-Muller, J. Y. et al. Aldehyde dehydrogenase 1a 3 defines a subset of failing pancreatic beta cells in diabetic mice. Nat. Commun. 7, 12631 (2016).

33. Demarchi, F., Verardo, R., Varnum, B., Brancolini, C. \& Schneider, C. Gas6 anti-apoptotic signaling requires NF-kappa B activation. J. Biol. Chem. 276, 31738-31744 (2001).

34. Loftus, J. C. et al. TROY (TNFRSF19) promotes glioblastoma survival signaling and therapeutic resistance. Mol. Cancer Res. 11, 865-874 (2013).

35. Hashimoto, T., Schlessinger, D. \& Cui, C. Y. Troy binding to lymphotoxinalpha activates NF kappa B mediated transcription. Cell Cycle 7, 106-111 (2008).

36. Hasanbasic, I., Cuerquis, J., Varnum, B. \& Blostein, M. D. Intracellular signaling pathways involved in Gas6-Axl-mediated survival of endothelial cells. Am. J. Physiol. Heart Circ. Physiol. 287, H1207-H1213 (2004).

37. Scutera, $\mathrm{S}$. et al. Survival and migration of human dendritic cells are regulated by an IFN-alpha-inducible Axl/Gas6 pathway. J. Immunol. 183, 3004-3013 (2009).

38. Moore, F. et al. STAT1 is a master regulator of pancreatic $\{$ beta $\}$-cell apoptosis and islet inflammation. J. Biol. Chem. 286, 929-941 (2011).

39. Mattisson, I. Y. et al. Elevated markers of death receptor-activated apoptosis are associated with increased risk for development of diabetes and cardiovascular disease. EBioMedicine 26, 187-197 (2017).

40. Ye, D., Ma, S., Xiong, Y. \& Guan, K. L. R-2-hydroxyglutarate as the key effector of IDH mutations promoting oncogenesis. Cancer Cell 23, 274-276 (2013).

41. Losman, J. A. et al. (R)-2-hydroxyglutarate is sufficient to promote leukemogenesis and its effects are reversible. Science 339, 1621-1625 (2013).

42. Mi, Y. et al. A novel function of TET2 in CNS: sustaining neuronal survival. Int. J. Mol. Sci. 16, 21846-21857 (2015).

43. Cull, A. H., Snetsinger, B., Buckstein, R., Wells, R. A. \& Rauh, M. J. Tet2 restrains inflammatory gene expression in macrophages. Exp. Hematol. 55, 56-70 e13 (2017).

44. Groom, J. R. \& Luster, A. D. CXCR3 ligands: redundant, collaborative and antagonistic functions. Immunol. Cell Biol. 89, 207-215 (2011).

45. Cardozo, A. K., Kruhoffer, M., Leeman, R., Orntoft, T. \& Eizirik, D. L. Identification of novel cytokine-induced genes in pancreatic beta-cells by high-density oligonucleotide arrays. Diabetes 50, 909-920 (2001).

46. Donath, M. Y., Boni-Schnetzler, M., Ellingsgaard, H., Halban, P. A. \& Ehses, J. A. Cytokine production by islets in health and diabetes: cellular origin, regulation and function. Trends Endocrinol. Metab. 21, 261-267 (2010).

47. Cardozo, A. K. et al. IL-1beta and IFN-gamma induce the expression of diverse chemokines and IL-15 in human and rat pancreatic islet cells, and in islets from pre-diabetic NOD mice. Diabetologia 46, 255-266 (2003).

48. Antonelli, A., Ferrari, S. M., Corrado, A., Ferrannini, E. \& Fallahi, P. CXCR3, CXCL10 and type 1 diabetes. Cytokine Growth Factor Rev. 25, 57-65 (2014).

49. Haase, T. N. et al. Growth arrest specific protein (GAS) 6: a role in the regulation of proliferation and functional capacity of the perinatal rat beta cell. Diabetologia 56, 763-773 (2013). 
50. Roep, B. O. Are insights gained from NOD mice sufficient to guide clinical translation? Another inconvenient truth. Ann. N. Y. Acad. Sci. 1103, 1-10 (2007).

51. Ligthart, S. et al. DNA methylation signatures of chronic low-grade inflammation are associated with complex diseases. Genome Biol. 17, 255 (2016).

52. Ansari, I. et al. The microbiota programs DNA methylation to control intestinal homeostasis and inflammation. Nat. Microbiol. 5, 610-619 (2020).

53. Le Floch, J. P., Escuyer, P., Baudin, E., Baudon, D. \& Perlemuter, L. Blood glucose area under the curve. Methodol. Asp. Diabetes Care 13, 172-175 (1990).

54. Ponath, G. et al. Myelin phagocytosis by astrocytes after myelin damage promotes lesion pathology. Brain 140, 399-413 (2017).

55. Steptoe, R. J. et al. Persistence of recipient lymphocytes in NOD mice after irradiation and bone marrow transplantation. J. Autoimmun. 22, 131-138 (2004).

56. Corces, M. R. et al. An improved ATAC-seq protocol reduces background and enables interrogation of frozen tissues. Nat. Methods 14, 959-962 (2017).

57. Buenrostro, J. D., Giresi, P. G., Zaba, L. C., Chang, H. Y. \& Greenleaf, W. J. Transposition of native chromatin for fast and sensitive epigenomic profiling of open chromatin, DNA-binding proteins and nucleosome position. Nat. Methods 10, 1213-1218 (2013)

58. Bolger, A. M., Lohse, M. \& Usadel, B. Trimmomatic: a flexible trimmer for Illumina sequence data. Bioinformatics 30, 2114-2120 (2014).

59. Li, H. \& Durbin, R. Fast and accurate short read alignment with BurrowsWheeler transform. Bioinformatics 25, 1754-1760 (2009).

60. Orchard, P., Kyono, Y., Hensley, J., Kitzman, J. O. \& Parker, S. C. J. Quantification, dynamic visualization, and validation of bias in ATAC-seq data with ataqv. Cell Syst. 10, 298-306 e294 (2020).

61. Zhang, Y. et al. Model-based analysis of ChIP-Seq (MACS). Genome Biol. 9, R137 (2008).

62. Quinlan, A. R. \& Hall, I. M. BEDTools: a flexible suite of utilities for comparing genomic features. Bioinformatics 26, 841-842 (2010).

63. Ross-Innes, C. S. et al. Differential oestrogen receptor binding is associated with clinical outcome in breast cancer. Nature 481, 389-393 (2012).

64. Heinz, S. et al. Simple combinations of lineage-determining transcription factors prime cis-regulatory elements required for macrophage and B cell identities. Mol. Cell 38, 576-589 (2010).

\section{Acknowledgements}

We thank Lesley Devine and Chao Wang for cell sorting. We thank Mei Zhong from Yale Stem Cell Center Genomics Core facility for RNA-seq library preparation and sequencing. This work was supported by grants DK057846 and DK045735 from the NIH, grant and SRA2014-142-S-B from the Juvenile Diabetes Research Foundation, a Pilot award from the Yale Diabetes Research Center P30DK116577, and a gift from the Howalt family. The Yale Stem Cell Center Genomics Core was supported by the Connecticut Regenerative Medicine Research Fund and the Li Ka Shing Foundation.

\section{Author contributions}

J.R. and K.C.H. conceived and designed the experiments and analyzed data. J.R. and S.D. conducted most of the cellular and animal experiments. A.L.P. acquired the human pancreatitis slides, prepared the MHCI tetramer for IGRP staining, and performed the human islets culture experiments. G.P. and M.L.-R. labeled and viewed the human pancreas slides with the supervision from D.P., R.K. and N.L. processed the samples for ATAC-seq and analyzed the data with the supervision from M.L.S. T.S. prepared RNA and cDNA amplification for RNA-seq libraries. M.L.S., D.P. and J.L. provided valuable inputs. J.R. and K.C.H. wrote the paper with input from all the authors.

\section{Competing interests}

The authors declare no competing interests.

\section{Additional information}

Supplementary information The online version contains supplementary material available at https://doi.org/10.1038/s41467-021-25367-z.

Correspondence and requests for materials should be addressed to K.C.H.

Peer review information Nature Communications thanks Defu Zeng and the other anonymous reviewer(s) for their contribution to the peer review of this work. Peer reviewer reports are available.

Reprints and permission information is available at http://www.nature.com/reprints

Publisher's note Springer Nature remains neutral with regard to jurisdictional claims in published maps and institutional affiliations.

Open Access This article is licensed under a Creative Commons Attribution 4.0 International License, which permits use, sharing, adaptation, distribution and reproduction in any medium or format, as long as you give appropriate credit to the original author(s) and the source, provide a link to the Creative Commons license, and indicate if changes were made. The images or other third party material in this article are included in the article's Creative Commons license, unless indicated otherwise in a credit line to the material. If material is not included in the article's Creative Commons license and your intended use is not permitted by statutory regulation or exceeds the permitted use, you will need to obtain permission directly from the copyright holder. To view a copy of this license, visit http://creativecommons.org/ licenses/by/4.0/.

(C) The Author(s) 2021 